\title{
Placing Location-Based Narratives in Context through a Narrator and Visual Markers
}

This is a pre-copyedited, author-produced version of an article accepted for publication in Interacting with Computers following peer review. The version of record "Valentina Nisi, Enrico Costanza, Mara Dionisio; Placing Location-Based Narratives in Context Through a Narrator and Visual Markers. Interact Comput 2017; 29 (3)" is available online at: https://academic.oup.com/iwc/article-abstract/29/3/287/2607835/Placing-Location-BasedNarratives-in-Context

DOI: https://doi.org/10.1093/iwc/iww020

\begin{abstract}
Location-based services, narratives and interactive experiences have a substantial tradition in $\mathrm{HCl}$. Building on this background, we report the iterative design process, prototyping and evaluation of Seven Stories, a location-based narrative experience. The design includes two novel features that aim to link the user experience more closely to its physical context: visual markers scanning, and a narrator. We have evaluated Seven Stories with two studies. Study 1, with 12 participants was conducted using qualitative methods to assess the overall user experience. Study 2, with 21 participants was conducted using quantitative methods, to evaluate the role of the markers and narrator as specific design features. Our contribution is to illustrate, through both qualitative and quantitative findings, how these two novel features influence the user experience, and what implications they have for design.
\end{abstract}

\section{Keywords}

Location-based Media; interactive narrative; GPS; visual markers; qualitative studies; cultural heritage; deployment, Information Interfaces and Presentation (e.g. $\mathrm{HCl}$ ): Multimedia Information Systems

\section{INTRODUCTION}

Urban spaces are an intertwined fabric of stories, memories, fears and hopes of past dwellers, current visitors and future perceptions. As such cities can be seen as cultural artefacts, a complex totality of cultural practices, old and new (Lynch, 1960; Stevens, 2007). New technologies are continuously developed to augment people, places, and settings, without necessarily addressing user-specific needs, but instead creating opportunities and encouraging different ways of behaving. A key concern for HCI today is how people react, change, and integrate these opportunities into their everyday lives (Rogers, 2011). Motivated by this perception of urban areas, as well as by the sensational spread of location-based applications and services, we engaged in a series of explorations and evaluations around the idea of augmenting the city with locally collected multimedia folk stories. This work can be ascribed to the subject of technology supported cultural applications. Today the study of such systems in the wild is gaining considerable attention in the $\mathrm{HCl}$ community (Rogers, 2011). $\mathrm{HCl}$ has extended its focus into these fields with an open mind and will reconsider its own tools in order to engage with more fluid and unquantifiable aspects of such artefacts (Benford, Giannachi, Koleva, \& Rodden, 2009).

Location-based services, narratives and interactive experiences have had a substantial tradition in $\mathrm{HCl}$. Over the last two decades many research efforts have explored the association of digital media with specific locations or objects. However, a large portion of the early literature has a technical focus - it reports systems and architecture demonstrating that location-based systems 
can be built (Abowd et al., 1997; Schilit, Adams, Gold, Tso, \& Want, 1993; Want, Hopper, Falcão, \& Gibbons, 1992) or frameworks and infrastructure created to enable potential applications (Angus et al., 2008; Hansen \& Grønbæk, 2008; Siira, Tuikka, \& Tormanen, 2009). Later contributions, especially around the theme of narratives, cultural itineraries, museum experiences and gaming (Brown et al., 2005; Crow, Davenport, \& Pan, 2003; Nisi, Oakley, \& Haahr, 2006), are generally reported in terms of user-experience case-studies or experience design guidelines for narrative and cultural applications (Nisi et al., 2006; Reid, Hull, Cater, \& Fleuriot, 2005).

Building on such a rich research background, this paper reports on the iterative design process, prototyping and evaluation of Seven Stories, a location-based narrative experience set on the island of Madeira. Through early assessment of the work we learned that our initial design fell short with regard to some crucial user experience issues, such as user orientation and engagement. Having identified these deficits, we introduced two new features with the aim of linking the user experience more closely with the physical location where it takes place in order to improve its continuity. In particular, we integrated the scanning of designable visual markers (Costanza \& Huang, 2009), to align digital content more precisely with physical locations, and framed the stories using a narrator as a literary device. The narrator introduces the stories to users and explains the link between the stories and their surroundings. It also helps users to find the best place to consume the digital content and it provides clues about the location of the next story. Finally the story is narrated in the video clip fragment. Seven Stories was evaluated first through qualitative methods using 12 participants to assess the overall experience. Subsequently a further quantitative study using 21 participants was conducted to evaluate the role of the markers and narrator as specific design features. Our contribution is to illustrate, through both qualitative and quantitative findings, how these two features influence the user experience, and what implications these have that go beyond the findings of previous studies (Fitzgerald, Taylor, \& Craven, 2013; Reid et al., 2005; Vriesede \& Nack, 2011). Our work also builds on the work by Petersen et al (Petersen, Iversen, Krogh, \& Ludvigsen, 2004), and in particular the concept of "Aesthetic Interaction" - the user experience is designed and evaluated paying attention to the aesthetic pleasure of the interaction. Petersen et al. highlights an aesthetic of use of interactive systems, which goes beyond the aesthetic of their visual appearance.

\section{RELATED WORK}

Location-based systems have been a topic of research since the early 1990 s when they were first introduced as part of the ubiquitous computing paradigm (Schilit et al., 1993; Want et al., 1992). The research conducted in this field is extensive and multifaceted. A substantial part of it, especially at the outset, was driven by the technological challenges that such systems pose. For readability we break the related work in several subsection although much of the work cited could fall under more than one sub section.

\subsection{Mobile Location Aware Guides}

Originally, systems like context-aware tour guides (Abowd et al., 1997) or generic infrastructures which link web resources to real-world locations (Hansen \& Grønbæk, 2008) have been proposed and built simply as proofs of concept. Pioneering and inspiring examples of locationbased guides include the work of Cheverst et al. (Cheverst, Davies, Mitchell, \& Friday, 2000) who developed "GUIDE", a mobile tour guide running on tablet computers connected to the internet through a Wi-Fi network. Similarly, but for indoor use, Ciolfi and Bannon built a museum guide to help visitors receive extra information about the artefacts encountered while roaming the collections (Ciolfi \& Bannon, 2007). Given that visiting museums and monuments can be a social activity, Aoki et al, designed SottoVoce (Aoki et al., 2002; Grinter et al., 2002) a system that augments the visit of an historic house without isolating the guests from each other. 


\subsection{Location Based Experiences}

In more recent years, as the importance of user experience has come to the foreground, the research focus in this area has shifted from the technical challenges to the ways in which designers can evaluate and refine location-based experiences in terms of immersion in the experience, flow, poetics (Millard \& Hargood, 2015) and aesthetic pleasure (Benford et al., 2009; Crabtree et al., 2004; Reid et al., 2005; 'Uncle Roy All Around You', n.d.). Along the same lines, Verplank highlighted how user experience designers can learn from the field of Urban Studies, taking into account the legibility of a physical space, such as the city (Verplank, n.d.). For instance, projects such as Grønland look at how to engage artists in creating location-aware poetry that can only be fully experienced in particular places of the city (Løvlie, 2012). However, while the work on Grønland is focused on the poets as content creators, in our work the emphasis is on the audience experience during Seven Stories. Projects such as Storystream strive to adapt and personalise the delivery of a corpus of stories to an audience on the move, taking into account users profiles and story context (Vriesede \& Nack, 2011). Despite authors recognising their system as being most suitable for documentary content, the lessons learned from the project proved useful in terms of system design and user preferences when experiencing mobile location-aware story material.

\subsection{Context Aware Storytelling and Gaming}

Narrative and cultural heritage have been, for sometime now, rich areas of application for location-based systems. Several projects have explored the potential of such technology as a form of context-aware story telling and gaming. Examples include many of the prototypes developed and tested within the "Mobile Bristol" and "Equator" groups of research projects, such as Riot1831, where public reactions to the mediascape are collected and distilled in a series of guidelines for designing location-based drama (Cater, Fleuriot, Hull, \& Reid, 2005); Fitgerald et al conducted further studies based on the initial experiments of the Mobile Bristol team and compared mobile location-aware audio tours with the same tour given by human tour guides. The findings highlight pros and cons for each mode of exploration. Flexibility and playback emerge as a plus for technology enhanced tours, while social bonding and human contact were most appreciated in the human-led experience (Fitzgerald et al., 2013). Another inspiring example of a story-driven location-aware experience is the "History Unwired" project (Epstein \& Vergani, 2006), which makes use of GPS sensing and Bluetooth technology to augment tourist experiences in Venice. The application accompanies the audience through a walking tour of Venice's most hidden neighbourhoods meeting local characters (in the form of pre-recorded interviews) in their natural settings. Complementing the "History Unwired" storytelling content, Angesleva and Parks designed a series of site-specific installations, where thermo-chromatic ink interventions come to life when Bluetooth activated devices come into range with them (Parkes \& Ängeslevä, 2007).

\subsection{Complementing GPS technology to ameliorate the user experience}

As GPS inaccuracies are reported to be negatively affecting the user experience (Benford et al., 2006; Crabtree et al., 2004; Nisi et al., 2006) other projects are aimed at leveraging the uncertainty embedded in the technical infrastructure to design ever seamless experiences for their audiences (Chalmers \& Galani, 2004) (Bell et al., 2006). For example GPS-based games: "Uncle Roy All Around You"('Uncle Roy All Around You', n.d.) as well as "Can u see me Now", mixing online with "on street" players make use of the location self-reporting of outdoor players to obviate the GPS inaccuracy (Benford et al., 2006).

In some cases, designers replaced GPS with other technologies, such as RFID tags, as in the case of PAC-LAN (Rashid, Bamford, Coulton, Edwards, \& Scheible, 2006). The work we present goes beyond simply replacing GPS by another technique. Instead we use the integration of two different location technologies and an analogue narrative device to create a unique narrative experience. The idea of augmenting GPS with a complementary location technique was mentioned by Ballagas et al (Ballagas, Kuntze, \& Walz, 2008) in their discussion of the initial 
evaluation of the "REXplorer" system (a location based game working with GPS alone). Such an idea resonates in part with some of the existing practices around "geocaching", as reported by O'Hara (O'Hara, 2008). Geocaching is a game that uses a combination of GPS and physical objects that need to be collected by players. However, while in geocaching the objects are just tokens to be collected to score, our work is different in that it explores how GPS and physical objects can be integrated into a narrative experience in combination with the literary device of a narrator.

O'Hara (O'Hara et al., 2007a, 2007b) reports the design and evaluation of a mobile phone based system to enhance the experience of primary school children visiting the London Zoo. The system provides media content about the animals to the children as they scan barcodes available near some of the cages. Similarly, Hopstory (Nisi, Wood, Davenport, \& Oakley, 2004) delivers a character-driven story experience inside Dublin's Guinness Hopstore. By interacting with special content loaded cardboard cats through an iButton ${ }^{1}$, the audience collects sequentially ordered story fragments around the space for later viewing. The experiences of both O'Hara and Nisi place an emphasis on the collection of location-based content for later viewing and reflection, as opposed to the immediate consumption, like Seven Stories does.

\subsection{Guidelines for designing Location Based Storytelling Experiences}

Finally, the Wi-Fi-based M-Views system (Crow et al., 2003), "Media Portrait of the Liberties" (Nisi et al., 2006) and Fattoria Mediale Amsterdam-based projects (Nisi, Oakley, \& Posthuma De Boer, 2010) document how urban settings and neighbourhoods can be augmented with multimedia stories and design guidelines synthesised for future mobile story designers. Such guidelines have been extensively used in the design of our system. More recently location based story games have been employed to entertain visitors as they are guided to learn about specific historical places and monuments of a city (Ballagas et al., 2008; Haahr, 2015) or engage families and the general public in highly social experiences (Lund, Coulton, \& Wilson, 2011) while exploring the surrounding topography. In all these examples the investigation was focused on qualitative aspects of location-based experiences, taking into account how location-aware technology needs to be designed and managed in order not to spoil the gaming or narrative experience for its users.

\subsection{Storytelling Platforms on Smartphones}

In the last few years, thanks to the popularity of smart phones and their app stores, two accomplished Mobile storytelling applications have been released on the market: 7 Scenes (Team 7scenes, n.d.) and HearUsHere (HearUsHere Team, n.d.). Both developed in Amsterdam, HearUsHere, is a location based nonlinear storytelling application which uses GPS coordinates along with iBeacon/Bluetooth devices to place sounds, with an adjustable volume radius, at a location or near an object. While HereUsHere supports audio-only story assets, 7 Scenes has a more complex but less flexible set up and photos as well as videos can be utilised to support the storytelling. While projects like these reinforce the relevance and the need for guidelines and usability studies of Locative Media we could not find any documented study or evaluation of the platforms.

\subsection{Summary}

All these projects encompass a very diverse set of features and structures; some are gamebased or goal-oriented (Benford et al., 2006), some collect information for later viewing and reflection (Nisi et al., 2004; O'Hara et al., 2007a), others target museums and historic tours (Abowd et al., 1997; Aoki et al., 2002; Cheverst et al., 2000; Ciolfi \& Bannon, 2007). Some of the

\footnotetext{
${ }^{1}$ An iButton is a microchip similar to those used in smart card but housed in a round stainless steel button of $17.35 \mathrm{~mm} \times 3.1 \mathrm{~mm}-5.89 \mathrm{~mm}$ in size
} 
projects described above make use of specific places to situate site-specific stories and help users to get to know the local communities and their opinions (Epstein \& Vergani, 2006; Nisi et al., 2006, 2010) while others make use of games to relay location-related information to their audiences (Ballagas et al., 2008; Haahr, 2015). Given the diversity of these systems it is difficult to generalize lessons for the design of our own system. Nevertheless they all enrich the panorama of culturally inspired location-based experiences, and have helped us in different ways in conceiving and designing coherent systems and journeys for our users-. Indeed, Benford et al. pointed out the importance of considering interactive experiences in terms of coherent journey or 'trajectories' (Benford et al., 2009). In summary, through the design and production of Seven Stories we envisaged being able to collect local folklore and make it available for tourists and visitors while walking, thus enabling them to become immersed in the old part of the city of Funchal.

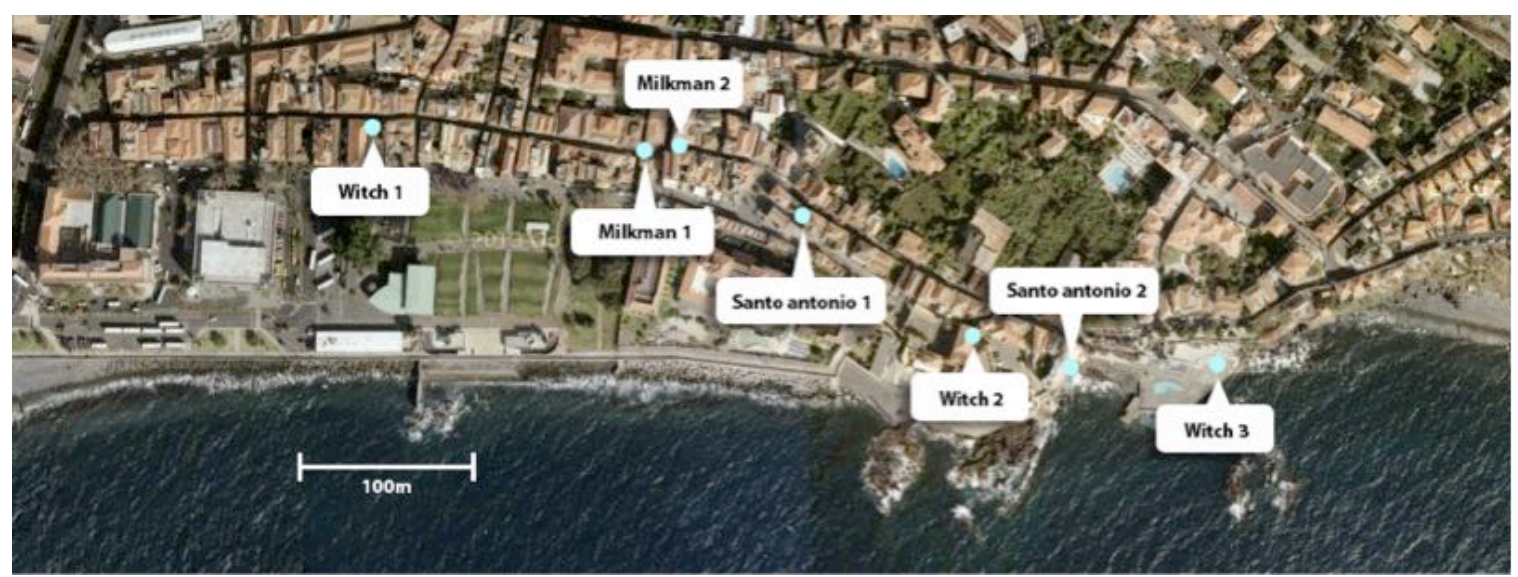

Fig. 1. The location where the experience took place, with indications of the stories placement (map: Google, SRES DRIGOT, DigitalGlobe).

\section{SEVEN STORIES: Background and Initial Content}

Madeira, a small island in the Atlantic Ocean, has a very rich traditional culture full of superstitions and folktales. In the past people would have had many opportunities to gather and share stories while nowadays opportunities for such sharing are becoming scarcer and some of this traditional culture is disappearing. Moreover the island is visited monthly by thousands of tourists who are not aware of its lore, albeit when offered information they are very interested in further investigation (van Leeuwen, Karnik, \& Keane, 2011). We saw this context as an opportunity to design and produce a location-based experience focused on local culture: a mobile application, which would treasure traditional folklore and make it available to locals, foreign residents and tourists. We also envisage our system functioning as a means of keeping the Madeiran culture alive so that traditional folk tales and local culture do not get lost over time. Our design process started from choosing the setting for the experience. To offer a contained experience, in a traditional setting, easy to navigate by locals as well as non-local people, we chose Rua Santa Maria in the town centre of Funchal, Figure 1. This particular street is located in the old part of town and it still maintains its traditional atmosphere, a suitable setting for the folk stories we wanted to deliver and ideal for the type of experience that we envisaged creating. Once the location was set, we researched old anecdotes and folktales from the local tradition to serve as the content for the narrative experience. Content was collected by means of word of mouth as well as some archived magazines and old paper clippings; then scripted and adapted 


\section{Seven Stories}

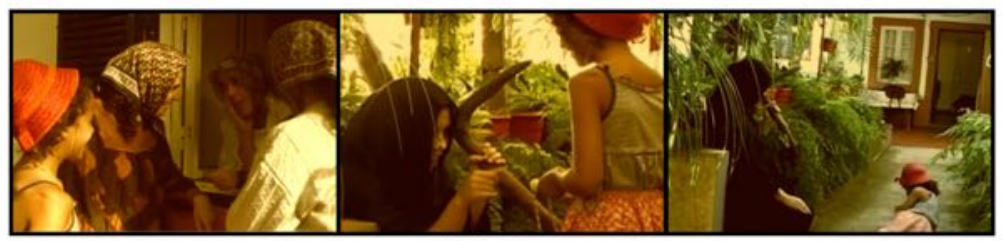

Witches 1
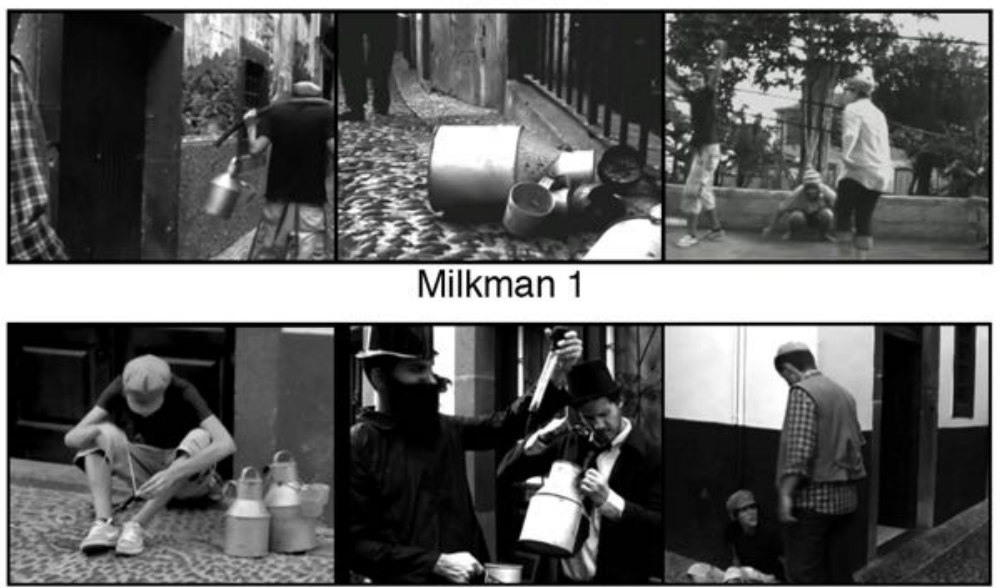

Milkman 2

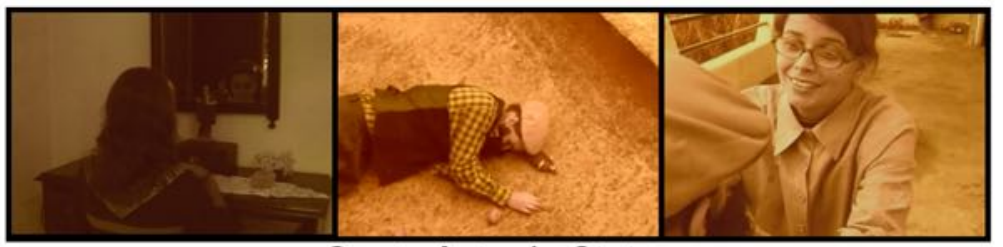

Santo Antonio Statue

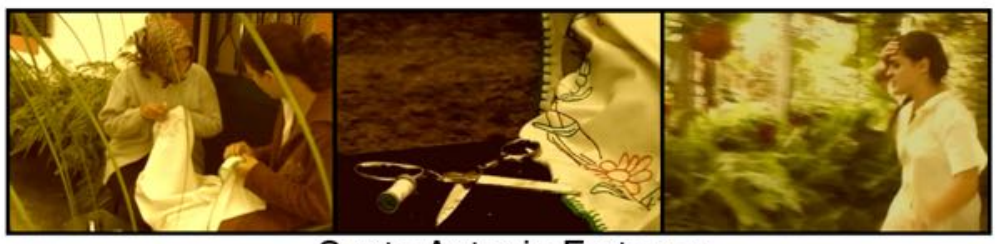

Santo Antonio Fortunes

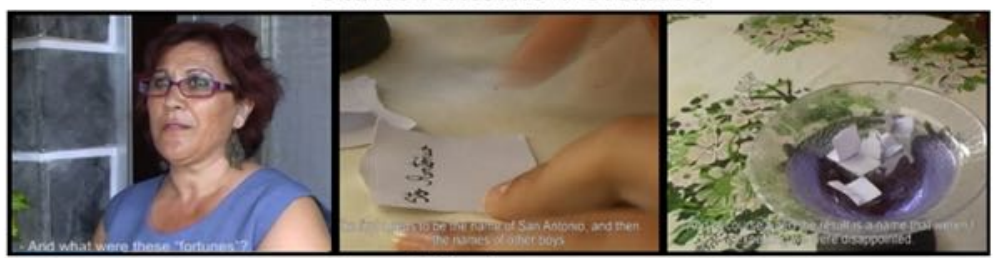

Witches 2

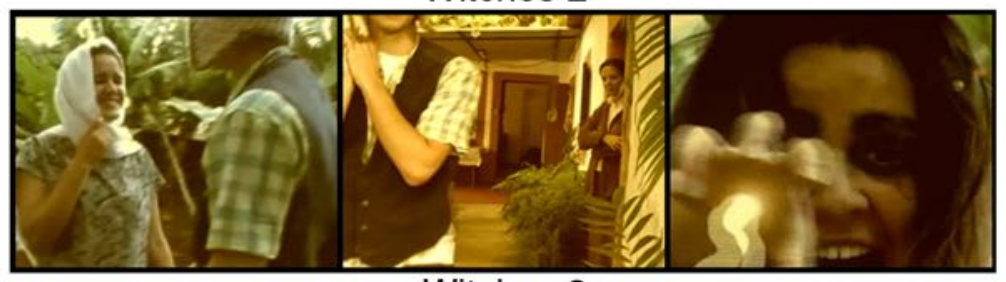

Witches 3

Fig. 2. Screenshots from the movie-clips of Seven Stories. 


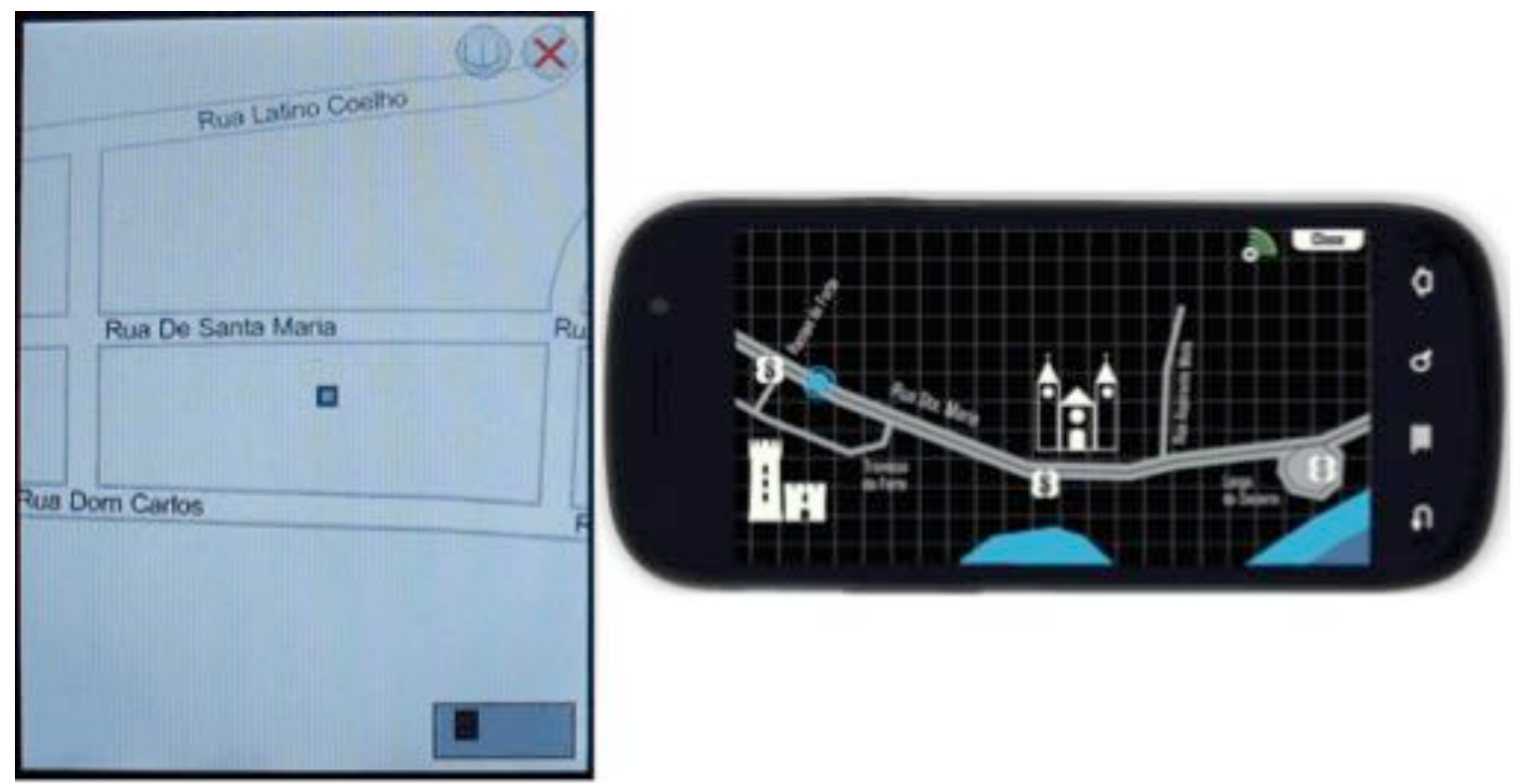

Fig.3. Screenshots of the interface. Left: version 1. Right: version 2

for multimedia production, paying attention to the size of the screen as well as to the duration constraints of a mobile experience (Crabtree et al., 2004). In order to create a connected journey through the narratives, the adaptation also emphasised links between the different story fragments. The video material was produced with amateur actors and took approximately four weeks to complete, including the final editing time. During this period seven short video-based stories were completed, with a duration ranging from one to three minutes each.

The audiovisual medium was chosen because it allowed us to render the traditional story plots, and complement them with further local traditional elements such as costumes, used objects and typical locations, such as interiors of houses and courtyards, embroidery practices, the local countryside, traditional milk cans and so on.

Three of the stories recount the adventures of a local. In the first of these three stories, Witch 1, we show some women gossiping about an old witch who lives nearby. The old witch approaches the young daughter on on of the women and transfers her magic powers into her just before passing away.. In the second story, Witch 2, two women are embroidering when suddenly the young witch girl from the first story, arrives. She has a reputation of being a witch, but she does not know it. The two women decide to use a trick to test whether the young girl is really a witch. The two women get their confirmation while the young girl remembers with horror her childhood incident with the old witch. In the last witch story, Witch 3 , the young witch who is no longer an innocent girl. This time, blinded by love, she decides to use her powers to conquer a married man with whom she has fallen in love.

The next group of two stories, inspired by real facts, focuses on a milkman, his daily routines, ups and downs and parental ménage. In Milkman 1, we follow the daily routines of a milkman and his son, who are working in the door to door distribution of milk.. In the next story, Milkman 2 , the milkman's son is in the street minding the cans of milk, when he is approached by two food inspectors, who analyse the milk and take the cans away for further inspection. Father and son end up teaming up against the inspectors in order to get their cans back.

Finally, two other stories are based on superstitious beliefs regarding the figure of Santo Antonio, the patron saint of couples. In the first of these stories, a very religious girl keeps on praying, year after year, for Santo Antonio to help her find a husband. As years continue to pass and she does not find a spouse, she throws the statue of the saint out of the window. A man who is passing by in the street below is hit on the head by the statue. It is so that she meets the love of her life. In the last story, "Santo Antonio Fortunes", we learn about the local ritual called 
"sortes" (which means "fortunes") .The story is about Maria, who gives her first hand testimony of how Santo Antonio and his fortunes helped girls find their future husbands.

Screenshots from each of the stories are shown in Figure 2. All of the stories have strong links to the locations in which the audience is able to retrieve them. Connections are made in particular through the visuals of the video material. For example, for the story where a Santo Antonio statue is thrown from the window of a balcony, we used an indoor setting for the video shooting, but a real window and balcony in the street are referenced by the narrator during the recounting of the story, to function as an anchor point in the setting where the audience experiences it.

\section{Introducing Narrator and Visual Markers}

A pilot study was conducted with five participants, as an initial evaluation of the Seven Stories experience in order to refine the interaction design to better cater for a more flowing and pleasurable experience. To enable the study an initial prototype was developed, as detailed in the following subsection.

\subsection{First Implementation}

The first implementation of Seven Stories was designed to be minimal in order to help the audience find the stories and to focus on the narrative experience. The main feature is a map where the locations of the stories are displayed as small dots, as illustrated in Figure 3 . The user position is marked on the map with a dot of a different colour. When the system detects that the user is within approximately 5 metres of a story location, the phone vibrates and the related story video clip can be played by pressing a button on the screen. The first version of Seven Stories was built on the Placeware framework (Nisi et al., 2010) a GPS-based location awareness framework that allows the presentation of multimedia videos. GPS was chosen over alternative location solutions such as beacons or Wi-Fi routers because it is self-contained in the phone, and in this way it limits the risk of damage to the infrastructure, due to weather or vandalism for example, given that the experience is set in a public space. To avoid network delays both the map and the videos are cached locally on the device.

\subsection{Gathering specifications through initial pilot testing}

Participants were initially asked about general demographics, familiarity with technology and with the city. Then the system was explained to them, after which each participant engaged in a 45 minute tour, shadowed by a researcher, and a 15 minute semi-structured interview. The interviews were aimed at investigating how users generally found the system, enjoyment of the experience and ease of use. Notes were taken during the shadowing and the interviews, these were later coded through open codes, and then grouped into categories.

Although the initial pilot was limited to five participants some patterns emerged very clearly from the data and prompted us to redesign the system, as described in the next section. The following paragraphs summarise the issues that we observed.

Engagement. We observed that all five participants positively engaged with the system and the content. They told us that they enjoyed the stories mostly because they could learn about the local traditions. Participants reported that they found the system easy to use, except for specific usability glitches (e.g. the dots indicating the stories on the map were too small), and the malfunction of the GPS, as we discuss next. Another comment frequently made was that the narrative was perceived as fragmented; this finding resonates with those from previous studies (Benford et al., 2009; Crabtree et al., 2004; Nisi et al., 2006; Rashid et al., 2006)..

Location Sensing and Orientation Issues. Participants were enthusiastic about the GPS tracking feature, and found the vibration signalling story content surprising and exotic. However, the technical limitations of GPS had a strong disruptive effect on the user experience. GPS 
simply did not work well in the narrow street so more than half of the location triggers were missed. Such limitation had been reported in the literature (Steed, 2004).

Orientation was generally reported as problematic for our participants. Even when GPS triggered the stories correctly, participants spent time looking around. During the interviews, they reported a lack of references in linking the story to its location. Moreover, in many cases participants were not sure about what direction to follow after viewing the clip, and in some cases they asked the researcher for instructions.

Landmarks and (Unfulfilled) Expectations. Two of the participants had strong and specific expectations that stories would be present near landmarks. Some places seem to naturally suggest to our participants that there would be a story attached to them so they stopped and waited even if the phone did not alert them to story content. It is worth emphasising that these location-based expectations emerged from both foreign and local participants.

Screen Capturing Attention. When participants were losing faith in the GPS functioning correctly they started focusing their attention on the device's screen, trying to find their way around using the map instead of relying on the vibrating function. Such focus on the screen partially defeated the object of our location-based experience, which was to acquaint participants with the architectural features and atmosphere of the old town.

\subsection{Design Iteration}

Based on the results of the pilot, we iterated the design of the system with the aim of producing a more cohesive and immersive user experience. In particular, the key issues that we aimed to address were the orientation problems, the visible disconnect between some of the stories and their physical context, and the reported feeling that the story fragments were disjointed. Therefore, we extended both the multimedia content and the interaction design of Seven Stories to include a narrator, and to integrate the scanning of visual markers to trigger the content playback. The following subsections describe each of these new features in more detail.

We decided to maintain the map-based interface, given that participants found it easy to use, but made it easier to read, redesigning the icons indicating stories and changing the colour scheme to cope better with outdoor daylight conditions, as illustrated in Figure 3 . The enthusiasm for the location-tracking (when it worked) and the vibration notifications indicating the proximity of a story motivated us to keep these features too.

\subsubsection{Narrator}

The use of a narrator is a well-identified narrative strategy, with the functions to contain and to frame. The narrator serves as a unifying container for collections of stories, which might otherwise be perceived as fragmented. Examples can be found in literary work such as Boccaccio's Decameron (1351-1353), Chaucer's 'Canterbury Tales' (1387-1400) or 'A Thousand and One Nights' (c.1450). The second function of the narrator, to frame, is used to give the reader a framework in which all the stories can be interpreted and understood. For example, in James's 'The Turn of the Screw' (1898), the whole story is narrated by a governess, who could be seen as being insane or as being a hero. In this example, it is left to the reader whether to interpret her as one or the other, and consequently how to interpret the whole story narrated by her (Abbott, 2008). This technique can have a powerful effect because readers can frame the whole experience according to the role and voice of the narrator.

We use both strategies to improve the user experience. Firstly, we use the narrator as a container in order to counteract the otherwise reportedly fragmented experience of the content of Seven Stories and to help users orient themselves in it. Secondly, by using a local character as the narrator we hope to enhance the perception or the illusion of the narrative being true stories that somehow happened to the local community in that street. The narrator was designed as a new and distinct character from the ones portrayed in the story fragments, modelled on a real historical figure from the neighbourhood where the experience takes place. This person was a local iconic figure who lived between 1920 and 2003. He owned a shop 
where the locals used to gather and share daily stories. We suggest that these are the stories that our narrator heard during his life and that he was now going to narrate to the audience through these special digitally mediated encounters.

The Seven Stories software application was modified so that when a user is in range of a story and the phone vibrates, an audio clip with the narrator's introduction to the story is automatically triggered. These introductory pieces were designed to direct users to where they could locate the story, and find the best place to watch the video clip, as well as to whet their appetites for the story that would follow. We decided to keep the narrator in audio format only to limit the demand for visual attention to the device screen while in between stories, especially since the narrator himself would verbally describe and point at features in the real environment to help users orient themselves and find the next piece of content. In this way we aimed at obviating the overwhelming feelings generated by having to find the story from its physical references in the real space and looking at the screen at the same time. For example, before one of the Santo Antonio stories, the narrator alerts users to look up to a certain balcony, as that same architectural reference will appear in the video fragment they are about to see and the story they are going to witness happened in that exact spot. At the end of the story, the narrator takes over again. He wraps up the story, reinforces some aspects of the narrative to better link them to the surroundings and sometimes prompts the audience to reflect on the moral of the story, the characters, or the costumes at the time. For example, in the Milkman story where a young boy spills all the milk in the irrigation channel to trick his father into believing that he has delivered all the milk he was given so he can play with his mates, the narrator prompts the audience to reflect on the double-sided meaning of that anecdote. On one hand the boy wasted all the milk while his father was relying on that profit, on the other, the young boy is just a child who wants to play with his friends but who already has a serious job and a lot of responsibility for his age.

Moreover, at the very beginning of the experience, when the application starts, the narrator helps the audience to understand how to use the system through an explanatory audiovisual clip introducing the system and its features to the users, explaining in detail how to use it, while at the same time familiarising themselves with its characters.
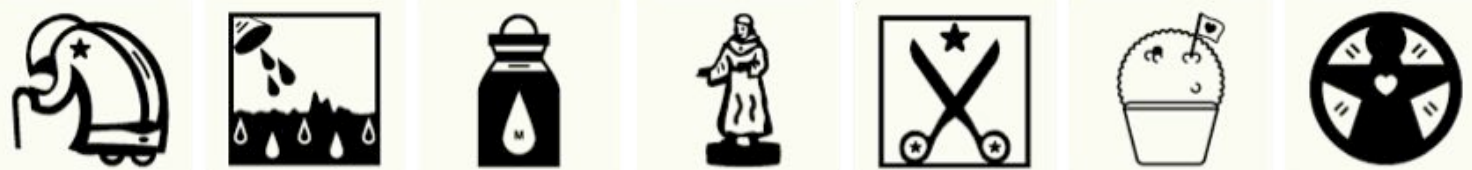

Fig. 4. The seven visual markers that correspond to the Seven stories. From left to right: Marker 1: related to the old witch -Marker 2: milk being spilled. Marker 3: the can of milk confiscated by the police. Marker 4: Santo Antonio statue. Marker 5: the pair of scissors part of the superstition about witches. Marker 6: a vase of the plant closely related to the Santo Antonio. Marker 7: a protective medallion related to the last witch story, and the protection of men against witchcraft.

\subsubsection{Visual Markers}

The system was modified so that the video clips were triggered by scanning visual markers through the smartphone camera, rather than by pressing a GUI button on the phone's screen. We decided to use the d-touch marker recognition system (Costanza \& Huang, 2009) because it allows the use of markers that can be visually designed and which hold semantic meaning for people (see for example Figure 4). The users of Seven Stories should find the markers attractive rather than seeing them as non-pictorial and meaningless glyphs.

Each visual marker indicates the location of a story. More precisely, we decided to place markers as close as possible to specific architectural features related to the content of the story, such as a door, a window or the corner of a building. Through their positions the markers are meant to create a bridge or link between the digital content and the physical place. The visual design of the marker is also related to elements of the story, to reinforce the connection. From the narrative point of view, the fact that the markers have aesthetic visual and semantic value is 
meant to help the audience to remain focused on the artistic value of the overall experience. For example, the marker for the story "The Statue of Santo Antonio" represents the statue, which is at the very centre of the narrative. The marker where the milk spills from the can into the water below depicts a scene from the story it is related to. More details can be found in the caption of Figure 4 . The markers are very inexpensive and easy to print, making it uncomplicated to replace them in case of damage.

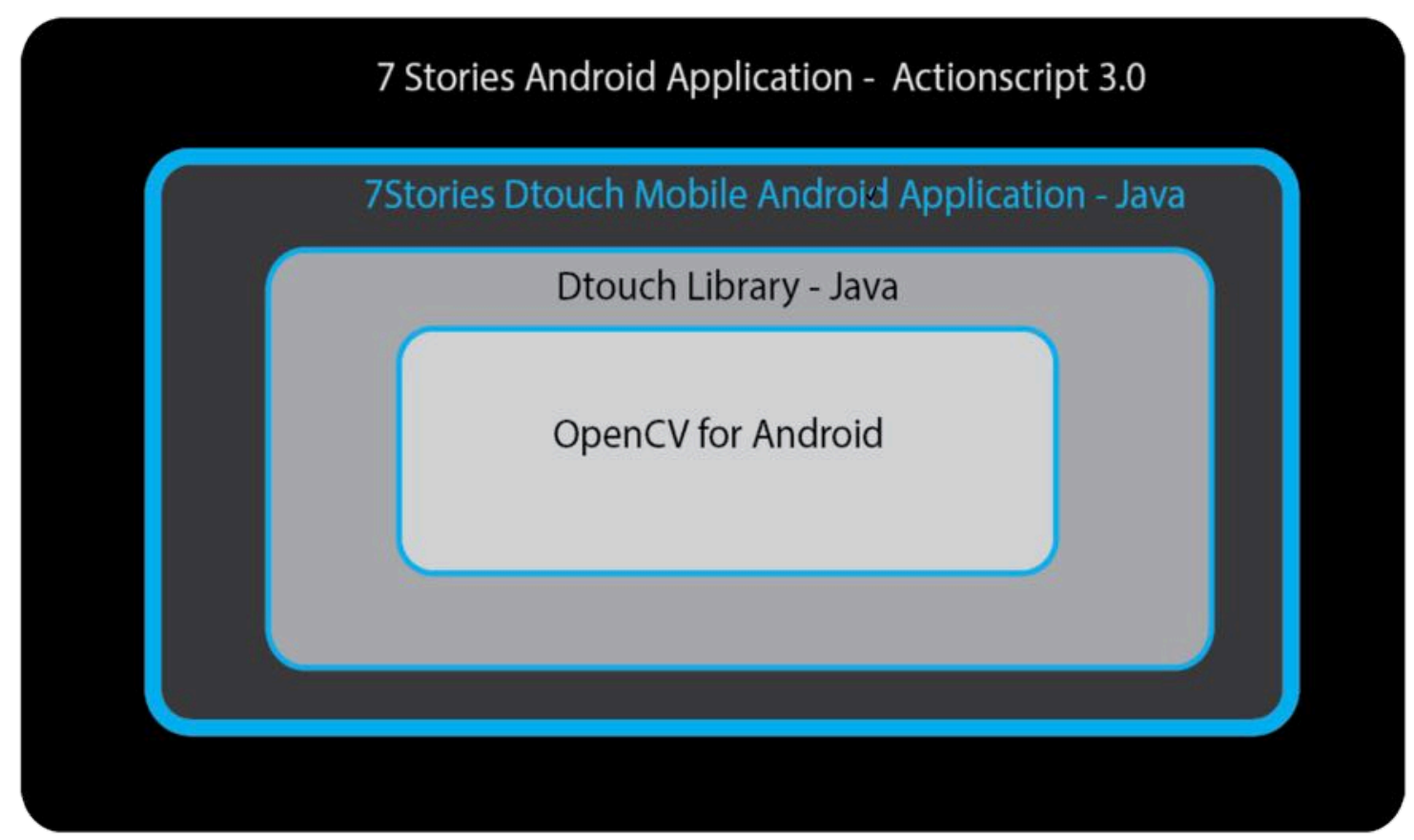

Fig. 5. Seven Stories mobile application modules

\subsection{Second Implementation}

From a technical point of view, after the initial pilot, the system was completely redeveloped to run on the Android platform. The Seven Stories application comprises several software applications embedded inside each other, as illustrated in the diagram in Figure 5. The main user interface of Seven Stories is developed in Flash Actionscript 3.0 and depends on the d-touch software in order to recognise the markers (Costanza \& Huang, 2009). The d-touch software system has a series of dependencies, namely the d-touch library and the OpenCV library (see Figure 5. for a software system diagram). To cater for our needs we adapted an open source Android version of the d-touch system developed at the University of Nottingham allowing it to communicate with our Actionscript application through a localhost socket. Moreover we adapted the d-touch system to read the markers that we designed by changing the levels of nesting originally allowed.

The main application of Seven Stories maintains all the content offline, which means that the map also works without Internet connection. For this we created an algorithm that translates the user position from the GPS coordinates into pixel coordinates, which allows us to display the user position as an icon on our graphic interface. The software was developed through an object-oriented approach. The Map object keeps verifying to see if the user position is overlapping with any story position. If so detected, it sends a message to the Stories object asking it to retrieve the ID corresponding to this story. Then another message is sent to the Narrator object requesting it to play the relevant audio clip. In this sequence of events, the vibration event is also triggered. Moreover the Map object is continuously listening for touch events happening on the story buttons; if the user presses a story button, the Map object is responsible for identifying which one was pressed. Then it will retrieve the information stored in 
the Stories object about the marker ID, the story ID and the video clip path so that it can send the parameters to the d-touch application via the localhost socket. From there it opens the dtouch application and plays the video if the ID sent from the main application is the same as the ID being read from the marker. The system was deployed on a Samsung Nexus S using Android 2.3.6.

\section{EVALUATION}

In order to test the refinements made to Seven Stories we evaluated the system through two iterative studies. The goal of Study 1 was to better understand the overall user experience, while in Study 2 we examined the effect of specific features of the system, through a comparative approach.

\subsection{Study 1}

A first in-depth qualitative study was designed and conducted in the field to evaluate the refined Seven Stories prototype. For the purpose of this study, and to provide a more realistic and practical scenario of deployment of this kind of technology, we set up collaboration with a local museum conveniently located near the starting point of the location-based narrative. Inside the museum, we showed participants an introductory video to provide instructions about the basic functioning of the system. This video was shown on the same device used for the rest of the experience, and lasted approximately 90 seconds. The video clarified all aspects related to the use of technology and its interface before the experience started, so that users would not feel overwhelmed by the amount of information they had to deal with during the stories. We shadowed participants as they walked and interacted with Seven Stories, and documented this observation through written notes and photographs. For each participant, the experience lasted between 30 and 40 minutes. Afterwards we asked participants to respond to a 30 question survey, taking approximately 20 minutes to complete. The questions explored how participants experienced the system and its content, including the relationship between stories and physical surroundings and the role of the narrator. For all participants the questionnaire was followed by a short semi-structured interview to discuss further specific aspects of the user experience, or issues the researchers noted as interesting during the observations. Interviews lasted approximately 10 minutes and were documented by the researcher who conducted them using handwritten notes.

\subsubsection{Participants}

Participants were recruited from the social network of the researchers. Twelve English-speaking users took part in the study: four locals and eight foreigners (three tourists and five residents). Six of the participants were professionals from various fields (real estate, veterinary, school teacher, software engineering) and six were university students from different disciplines (Human Computer Interaction, Design, Psychology). The sample included six women and six men, age range 20 to 41 years old.

Three of the participants described themselves as very comfortable with using technology, seven as comfortable and one as not comfortable. Ten of the users were familiar with locationbased systems like, for example, the ones used for orientation in cars but only four had used a location-based system for entertainment purposes or location-based systems in museums. Users were left free to decide whether to experience the system individually or in pairs. Four users went out in two pairs using a phone per couple, and the rest experienced the Seven Stories individually. It should be pointed out that participants were allowed to take part in pairs simply for practicality, and in the following results we do not distinguish between individual participants and pairs, as such differentiation is beyond the scope of our work. 


\subsubsection{Results}

We used thematic analysis (Braun \& Clarke, 2006) to identify themes and patterns in the data and the following main themes emerged: engagement, orienting function of the narrator, and markers as tangible feedback and reward. We will report on these three themes in the following subsections.

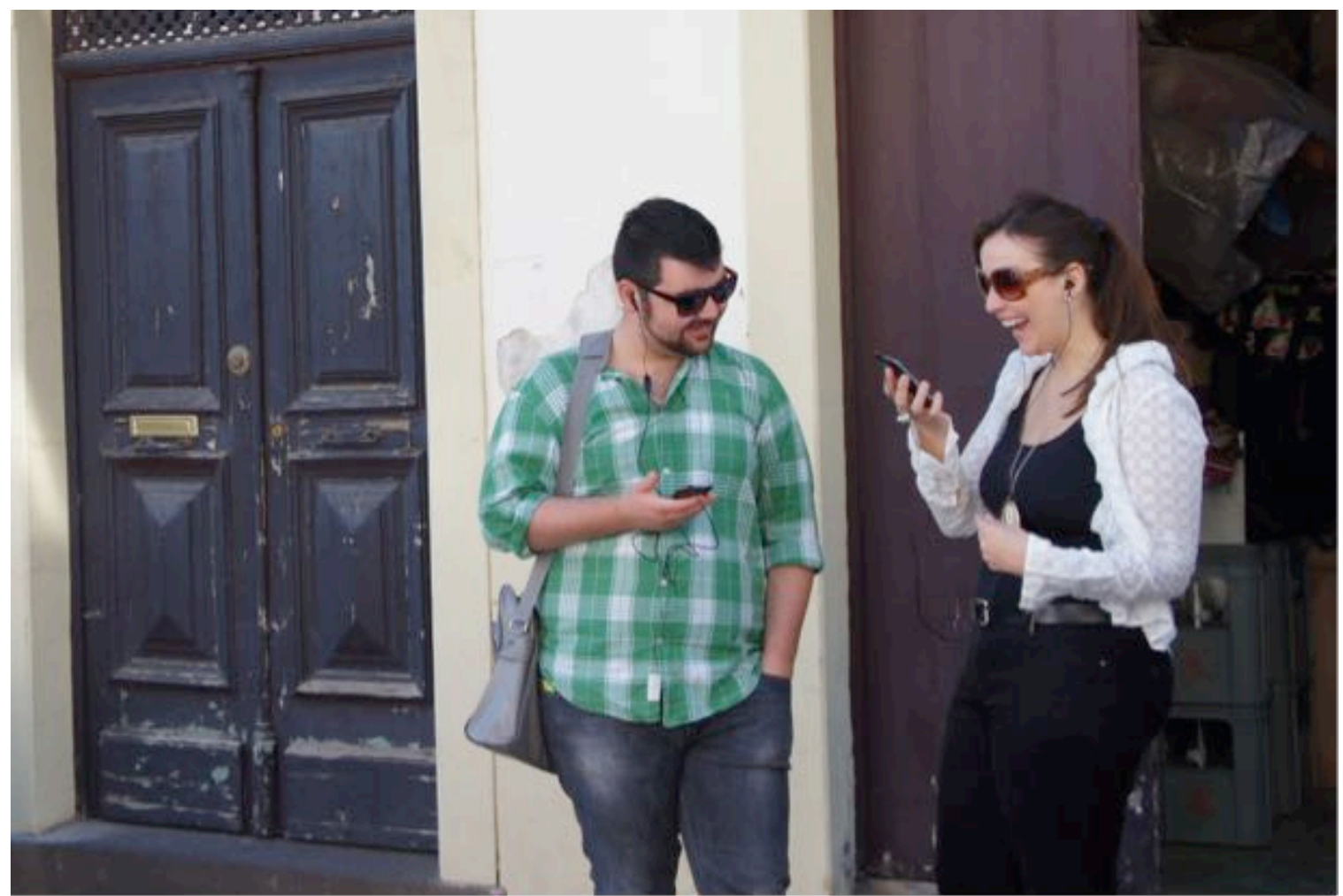

Fig. 6. A photo captured during Study 1, showing two participants enjoying the content of the Seven Stories experience.

\section{Engagement.}

Since the state of immersion or engagement of a participant in a game or narrative experience can be measured though the user awareness of time passing (Timothy Sanders, 2010; Wood, Griffiths, \& Parke, 2007) we asked participants about their perception of the duration of the experience. We found that seven out of the twelve users thought the overall experience had the ideal duration, four users wished that the experience could have been longer and only one user expressed that it could have been shorter. We interpreted these positive signs as an indication that we had crafted an experience that encouraged immersion or engagement and a pleasurable passing of time with our application. Furthermore, to support our interpretation, some users expressed the desire to have more stories and other places to explore. Lucas said: "I will be waiting for new stories here or in another street." Along the same lines Fernanda wished for an extension of the experience: "Maybe we could receive an invitation to see more stories online or in another street."

When asked what they would take away from the experience, most participants said that the stories were what they cherished the most. One of the foreign participants told us: "I learned about Madeira's folktales and enjoyed it." One of the local participants told us: "The stories! They were all new to me!" While another local participant added: "it was cool to remember stories from my childhood [...] I remember my mother telling me some of the fortune-telling acts performed during Santo Antonio festivities" As mentioned in the pilot study, the fact that participants reported such engagement with the stories suggests that the system essentially 
worked as a successful cultural application, and our measures of improvement (adding markers and the narrator) considerably smoothed the feeling of being overwhelmed and lost, and the audience could experience and enjoy the content more fully. Such insight is strengthened by our observations: as participants were watching the stories, it was possible to see the enjoyment in their facial expressions as illustrated for example in Figure 6.

As a further indication of engagement, we observed that the stories promoted conversation and exchange of further stories with our participants. For example one pair of participants discussed the popularity of Santo Antonio in different countries and cultures, while the other pair started discussing witch folktales from their own culture. Another participant told the researchers that some of the folktales in Seven Stories were similar to the ones from their own country and started describing them to us.

\section{Orienting Function of the Narrator}

Our observations suggest that the narrator was effective in orienting participants on most occasions. We noticed that the phone vibration worked almost like a command: users immediately stopped walking for a couple of seconds, they appeared alert, almost tense; then, as soon as the narrator started talking, they relaxed and started walking and looking around again. We also observed some participants nodding their heads when the narrator pointed them to the location where the story happened, as if to signify "yes we can see what you are referring to". We could observe the curiosity lighting up their faces, as they seemed very focused on searching for the marker. Another indication of the effectiveness of the narrator was provided by Lucio when answering a question about how to improve the experience he said: "more stories should make explicit use of the surroundings (through the narrator) like in the story of the Santo Antonio statue, when the narrator says: "Look up! That's the balcony from where the statue fell." On one hand this points out that when used, the narrator works well in orienting the users as to where to look and where to find connections with the environment. On the other, it reveals that it could have been used even more broadly than it was.

At the same time, we also gathered evidence of the persisting GPS failures and of the nature of the narrator's limitations as an orientation stratagem. First, when asked about any problems they had encountered in using the platform, four users mentioned the GPS, either regarding the long fixing time or inaccuracy. Second, as an improvement to the system, one user suggested adding a kind of compass that would help to navigate the map and point out to the users which direction to follow to find the next story. Clearly, for this user the narrator was not enough of a solution. Another issue that emerged in the study was that the narrator's indications partially rely on the user being close to the story hotspot, otherwise it becomes harder to make sense of his guidance. For example Manuela, after walking away from the marker, approached the researcher and asked: "Where is this window that the narrator mentions?" The window was, in fact, the one just beside where the marker was placed, but since the user kept walking forward while listening to the narrator talking, she missed this connection.

\section{Markers as Tangible Feedback and Reward.}

We observed that often when participants found a marker they experienced a feeling of reward or joy, as they smiled or nodded - finding a marker became almost a treasure hunt experience in itself. Sometimes a marker was spotted before the narrator indicated it, in this case participants seemed even happier when they found it before the narrator guidance, perhaps because they felt smarter than the system, because they could find the marker without the narrator's help. For example João turned to the researcher and pointing to the marker shouted with some excitement: "I know there's one [story] here right?" Similarly, when Nuno found a marker before getting the hint from the narrator, he turned to the researcher and said: "I discovered the marker! Now I just need wait for the narrator." As described above, the narrator audio clips are triggered by GPS, so if a marker is found without the narrator's help, it is usually because the GPS did not work or was too slow in getting the fix. We noticed, that sometimes the marker could remedy these technical issues: participants would see the marker and wait for the GPS to update their 
position. Participants were actually very patient in relation to the time that the GPS would take to update, perhaps because of the trustworthy feeling of the physical marker-object. We observed that sometimes they would take the waiting time as an opportunity to look around and enjoy the street surroundings.

While we shadowed our participants we also sometimes observed that passers-by, people who were not participating in the user study, also noticed the markers in the street and often showed curiosity towards them. Some passers-by asked us, the researchers, what the markers were for. We observed two teenage girls passing in front of Marker 7, illustrated in Figure 4., and one of them pointing to the other: "look at that drawing: it's cute!" On another occasion, we witnessed some elderly local ladies, who usually spend the afternoon sitting in the street chatting, or embroidering, discussing the marker. The ladies picked up from the floor some markers that fell and kept them safe, considering them something to be valued. They then returned the markers to us when they saw us looking for them in the street. Clearly the markers attract attention -we believe this characteristic of the markers is very positive in terms of participant enjoyment and creating general awareness in the area regarding the project and this can be exploited further. The marker recognition system also manifested its limitations. At least three users experienced difficulty in capturing markers, and as a consequence two of them exited the Seven Stories software application and turned to the phone default camera application to try and capture the marker. In another case, we observed how physical landscapes could be more attractive than the markers. In the introduction of the "Santo Antonio Fortune" story, the narrator refers to a church located 20 meters ahead. As soon as this is mentioned, three out of the twelve participants immediately directed themselves towards the church, ignoring the narrator saying that the marker was actually somewhere else.

\subsubsection{Discussion}

The results of Study 1 indicate that overall the Seven Stories experience was largely successful. We observed participants as being engaged in the experience, they seemed to appreciate the guiding function of the narrator, and considered the markers as a tangible feedback and reward. No feelings of being overwhelmed or lost were observed or reported. Participants were engaged and appeared to appreciate the features added to the system after the pilot. In particular, the integration of the narrator and the visual markers seems critical for making the experience overall successful.

The adoption of d-touch markers seems to largely compensate for GPS glitches: seeing the markers provides users clear, tangible feedback that they are in the right place. It also seems that that the markers have the potential to balance between the physical and digital elements of the experience. Markers highlight details of the physical space, before audiovisual clips are played. Using a narrator seems to support users in the exploration of the narrative space and physical environment, and helped in delivering a more satisfying and contextualised locationbased experience to our audience.

However, these two features were not the only differences from the initial version tested in the pilot: as described above the phone hardware was different, the visual design of the $\mathrm{UI}$ was also carefully redesigned to match the experience. A new question, then, emerged from Study 1: To what extent do markers and narrator contribute to the success of the user experience? To address it, we designed a second study, described in the next subsection.

\begin{tabular}{|c|c|c|c|}
\hline & GPS-only & Markers+GPS & Full \\
\hline Map & $\checkmark$ & $\checkmark$ & $\checkmark$ \\
\hline GPS-triggered vibration & $\checkmark$ & $\checkmark$ & $\checkmark$ \\
\hline Scan marker to trigger content playback & $x$ & $\checkmark$ & $\checkmark$ \\
\hline Narrator & $x$ & $x$ & $\checkmark$ \\
\hline
\end{tabular}

Table 1. Summary of the features of the 3 applications compared in Study 2 


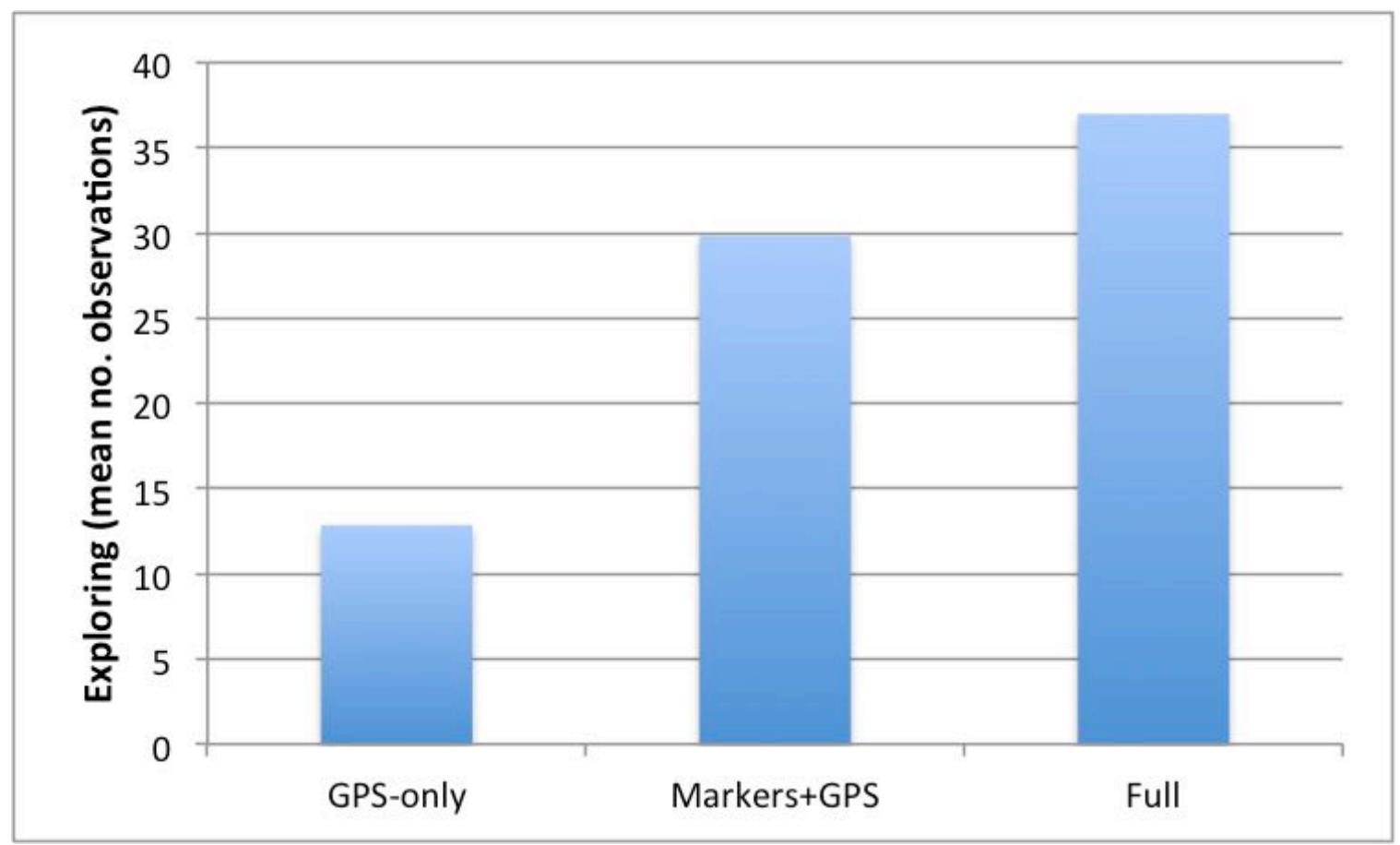

Fig. 7. Graph of the mean values of Exploring for each of the system versions.

\subsection{Study 2}

A second quantitative study was designed and conducted to evaluate how specific features of Seven Stories influence the interaction. To achieve this goal, we developed two simplified versions of the system and compared them through a between-subjects study to the full version of Seven Stories. The first simplified version uses only the GPS position information to let users access the stories, no narrator and no markers - we refer to this version as "GPS-only". The second simplified version uses both the GPS information and the d-touch visual markers, but no narrator - we refer to this version as "Markers+GPS". Table 1. highlights the different features available in each version. Each application version defined one of the three conditions of the study.

Given that the full version of Seven Stories was evaluated in Study 1, for practicality in Study 2 we modified the location of one of the stories, Witch 1, to make the overall experience shorter. The story was moved to a location a couple of meters before the Witch 2 story. As with Study 1 , we started by showing participants an introductory video explaining the functionality of the system.

An observation protocol was defined to document in a quantitative fashion participant behaviour. In particular one researcher recorded the following dependent variables for each user:

- $\quad$ How many times they go back and forward when looking for a marker;

- How many times they explore the surroundings, later referred to as "exploring" for brevity;

- $\quad$ How many times they seem lost;

- $\quad$ How many times they look to the researcher (to try and get help);

- $\quad$ Amount of time spent looking at the screen;

- $\quad$ Total duration of the experience.

The amount of time each participant spent looking at the screen, and the total duration of the experience were measured with the aid of a stopwatch. Beyond this observation protocol, the researchers kept written notes about anything else they witnessed which was related to the Seven Stories experience. 
At the end of the Seven Stories experience, we asked participants to complete a 30 question survey, which took approximately 10 minutes. Of the questions, 22 were based on 5-point Likert scales, and assessed how participants experienced the system, their engagement with the experience and its content, including the relationship between stories and physical surroundings. Nine of these questions were from the Flow Short Scale on absorption (Magyaródi, Nagy, Soltész, Mózes, \& Oláh, 2013). The remaining eight questions asked users about their background and their prior experience with technology and location-based systems.

\subsubsection{Participants}

Participants were recruited using snowball sampling. Twenty-one English-speaking users took part in the study: seven participants per each of the three conditions. In terms of demographics, we had thirteen females and eight males; eleven were from the island, while the other five were foreign residents, having lived on the island for between five and fifteen years. Five were foreigners who had recently arrived and had been visiting the island for less than a week. The average age of participants was $\mathrm{M}=28.7(\mathrm{SD}=6.3)$ ranging between 20 and 45 years old.

Sixteen of the participants described themselves as very comfortable with using technology, four as comfortable and one didn't answer the question. All participants owned a smartphone. Seven of the participants had used an application similar to Seven Stories, only two specified which (Geocaching application and museum application that delivers information on paintings).

Ten of the participants opted to take part in the experience in pairs. In this case, each participant was provided a phone and shadowed individually by one researcher. The pairs were distributed across conditions in the following way: two pairs for the GPS-only condition; two pairs for the Markers+GPS condition; one pair for the Full condition. As in Study 1, participants were allowed to take part in pairs just for practicality, and our analysis does not focus on the differences between pairs and individuals, as that is beyond the scope of our work.

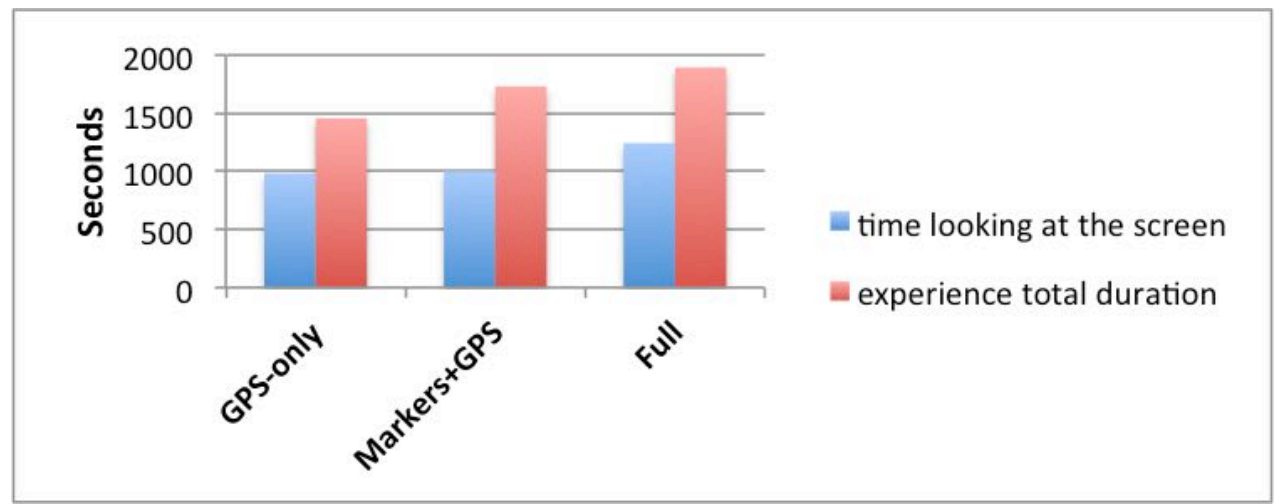

Fig. 8. Graph of the amount of time in seconds of Looking at the screen (left bar) and the Total duration of the experience (right bar) for each of the system versions.

\subsubsection{Results}

A one-way ANOVA revealed a significant effect of the conditions on exploring $(p<0.01$, $\mathrm{F}=8.843)$, time looking at the screen $(\mathrm{p}<0.01, \mathrm{~F}=14.672)$, and total duration of the experience ( $\mathrm{p}$ $<0.01, \mathrm{~F}=24.404)$. A post-hoc Tukey test revealed that exploring was lower in the GPS-only condition (average: 12.86) than in the other two conditions (Markers+GPS average: 29.86; Full average: 37.00 ), see Figure 7. A post-hoc Tukey test on time looking at the screen revealed that this variable was on average higher in the Full condition (mean: 1242.71, approx. 20' 43'), than in the GPS-only (mean: 984.43s, i.e. approx. 16' 24') and Markers+GPS (Mean: 1001.29s, i.e. approx. 16 $\left.41^{\prime \prime}\right)$ conditions. A post-hoc Tukey test on total duration revealed that the experience lasted less time in the GPS-only condition (mean: 1457.14s, i.e. approx. 24' 17') than in the Markers+GPS condition (mean: 1731.43, i.e. approx.: $28^{\prime} 51^{\prime \prime}$ ) and that in turn lasted less time than in the Full condition (mean: 1894.29, i.e. 31' 34'"), as shown in Figure 8. 


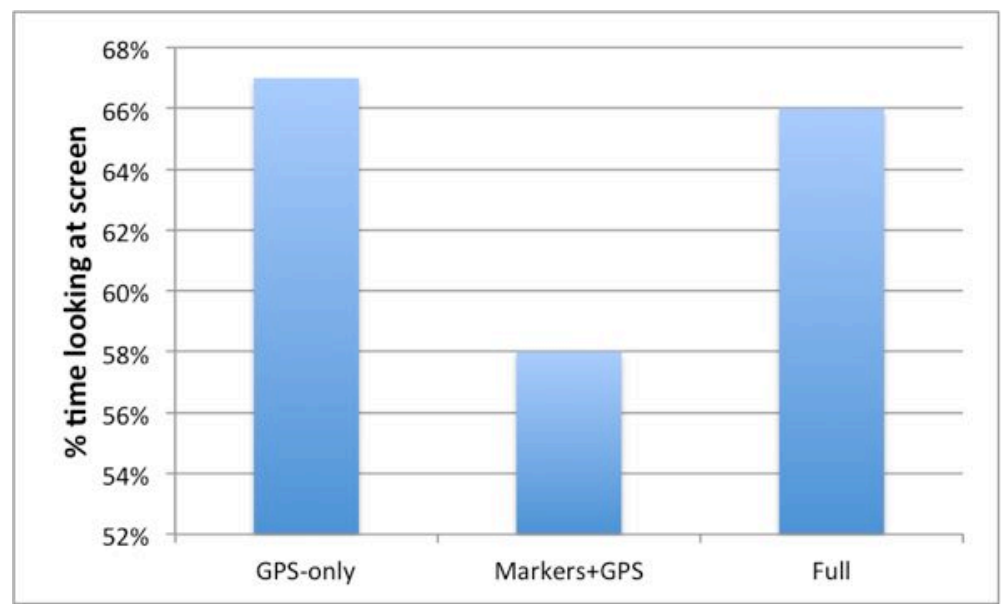

Fig. 9. Graph for the percentage of Time looking at the screen.

Given that the total duration of the Seven Stories experience varied for each participant, we analysed the amount of time participants looked at the screen as a percentage of the total duration of the experience. A one-way ANOVA revealed a significant effect $(p<0.01, F=6.275)$, and post-hoc Tukey test revealed that the percentage was on average lower in the Markers+GPS condition (mean: 58\%) than in the other two conditions (GPS-only mean: $67 \%$, Full mean: 66\%), see Figure 9. No statistically significant differences were found in the other observation variables. For these results a Levene's test revealed a significance value of greater than .05 , indicating that assumption of homogeneity of variances is not violated. Moreover we tested for the internal reliability of the absorption scale derived from flow short scale but its internal reliability was $a<0.7$ therefore results for the scale in general are not reported, nor the individual items.

Regarding the other Likert scale items, the Kruskal-Wallis Test revealed a significant effect of the conditions on two items: I found the experience of Seven Stories engaging $\times 2(2)=6.840, p=$ 0.033 ; I found the stories clearly related to the physical surroundings $\mathrm{X} 2(2)=11.027, \mathrm{p}=0.004$. No significant effects were found on the other Likert scale items.

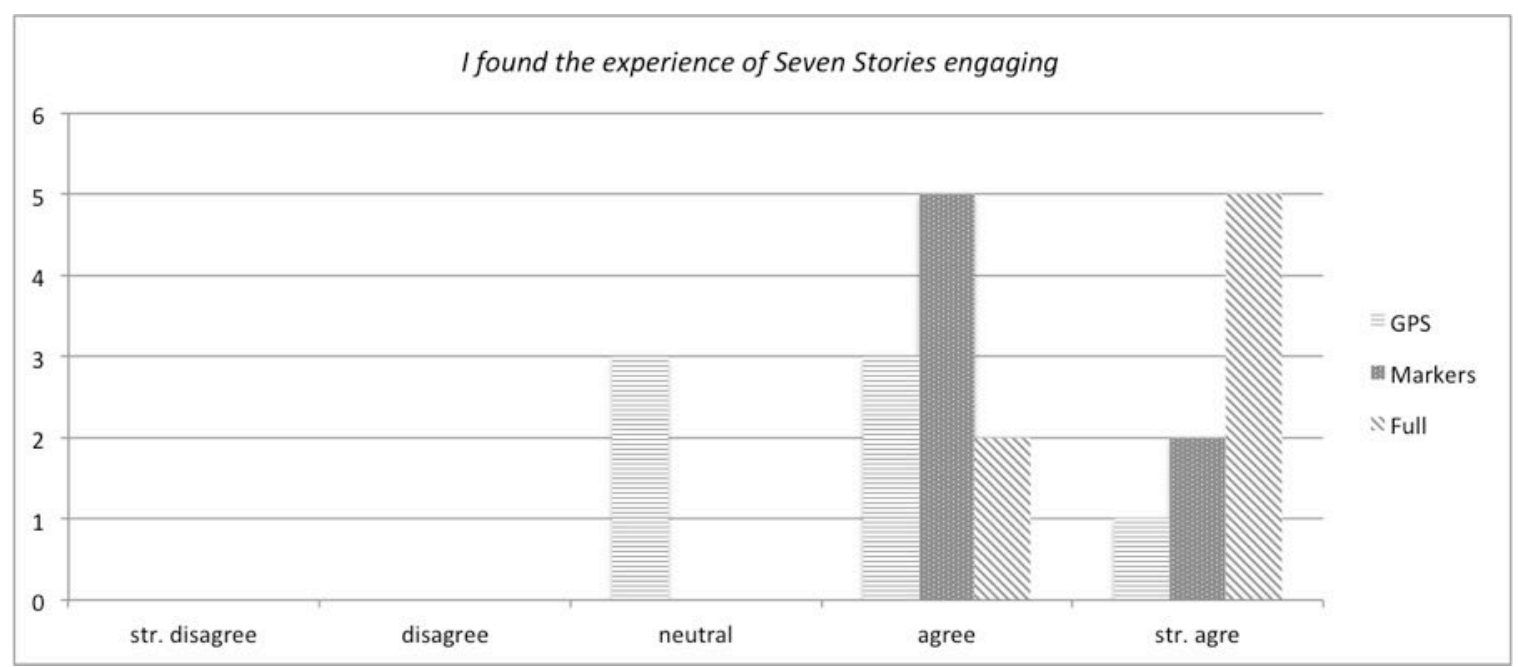

Fig. 10. Histogram of the responses to the question "I found the experience of Seven Stories engaging." The Kruskal-Wallis test and Mann-Whitney tests revealed that the GPS-Only condition is significantly different (lower ratings) from the Full condition. 


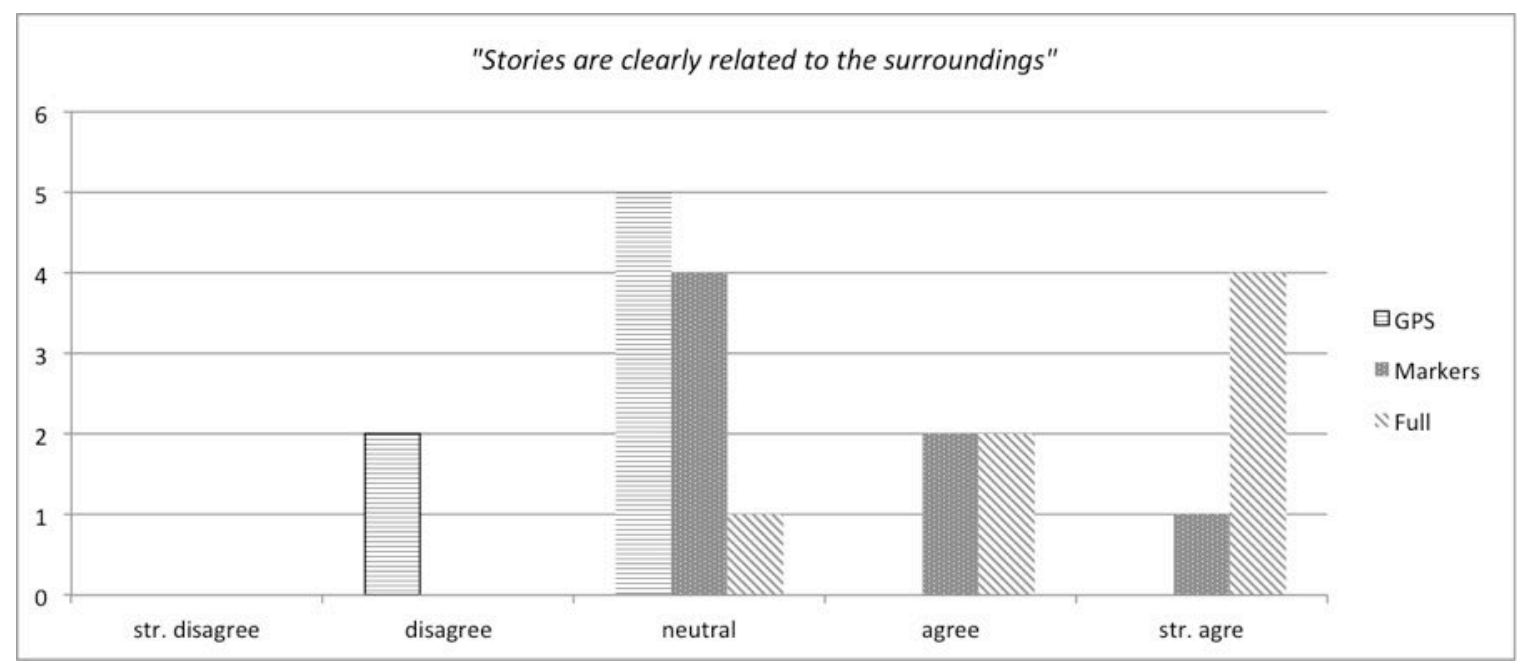

Fig. 11. Histogram of the responses to the question "Stories are clearly related to the surroundings." The Kruskal-Wallis test and Mann-Whitney tests revealed that the GPS-Only condition is significantly different (lower ratings) from the other two conditions.

Mann-Whitney tests were performed pair-wise on the three conditions, to identify which differences are significant. These tests revealed that engagement was rated lower in the GPSonly condition (mean rank: 5.07 ) than in the Full condition (mean rank: 9.93) $(U=7.5, Z=-2.33$, $\mathrm{p}=0.02$ ); while the Markers+GPS was not found to be significantly different from either of the other two.

The same tests revealed that participants rated Stories are clearly related to the surroundings significantly lower in the GPS-only condition (mean rank=4.36) compared to the Full condition (mean rank=10.64) $(U=2.5, Z=-2.96, p=0.003)$. The tests revealed also that the same question was rated significantly lower in the GPS-only condition (mean rank=5.43) compared to the Markers+GPS condition (mean rank=9.57) $(U=10, Z=-2.17, p=0.03)$. Instead no significant differences were found between the Markers+GPS condition and the Full condition. Figures 10 and 11 illustrate the responses for two likert items on which statistically significant differences were found.

\section{Additional observations.}

In the two conditions without narrator, GPS-only and Markers+GPS, at the end of the experience participants asked about the connection of the stories with the physical surroundings. This was observed four times in the GPS-only condition, and three times in the Markers+GPS condition, but never in the Full condition. Moreover, in the two conditions without narrator, participants demonstrated some confusion about the start and end of the experience (but never in the Full condition). In three of the GPS-only sessions and two of the Markers+GPS sessions participants where not sure about what to do after listening to the introduction video. Moreover, in two of the Markers+GPS sessions and three of the GPS-only sessions participants approached the researcher to ask whether the experience was over.

It was also observed that in the majority of instances the GPS-only version of the stories were not watched in their intended location. Participants stopped as soon as they felt the phone's vibration, but because of GPS errors this was sometimes the incorrect location. As a consequence, they often did not know where to stand while watching the stories, and sometimes they even stopped in potentially dangerous places, such as in the middle of the street (in these cases the researchers made sure that no danger was actually present or asked the participants to move).

With the Full version participants tended to look at the screen of the phone after hearing the vibration. Then while the narrator was speaking they would switch back and forth rapidly 
between looking at the screen and looking at the surroundings while trying to identify the features highlighted by the narrator.

Finally, similar to observations made in Study 1, we noticed that the markers attracted the attention of local people who did not take part in our study. In total five people, including two teenagers passing by, one local resident, and two people working in a local restaurant asked us about the markers.

In summary, the results of Study 2 revealed a number of differences across the three conditions. In terms of quantitative data, differences were found both on three of the measured variables (exploring, time looking at the screen, and total duration of the experience) and on two of the questionnaire items (I found the experience of Seven Stories engaging, and I found the stories clearly related to the physical surroundings). Differences were also noticed through the qualitative observations, which suggested that the markers and the narrator had a positive effect on the user experience.

\subsubsection{Discussion}

The analysis of the "exploring" variable derived from the observation further explains the role of visual markers in the user experience. In the GPS-only condition exploring was significantly lower, compared to the other two conditions, which integrate d-touch markers in the system. A possible explanation, is that the activity of looking for the visual markers encourages users to explore the physical surroundings. It is worth noting that the average number of observed "explorations" (ranging from 12.86 in the GPS-only condition to 37.00 in the Full condition) does not correspond directly to the number of markers in Seven Stories (just 7): the exploration activity goes beyond just looking for the markers.

The result for "amount of time looking at the screen" indicates that no statistically significant differences were found between the GPS-only and the Markers+GPS conditions. In contrast, in the Full condition participants on average looked at the screen for longer than in the other two conditions. These results can be explained as follows: in all cases participants looked at the screen when the stories play - this amount of time is constant for all conditions. Moreover, in all conditions participants looked at the map to navigate. In the GPS-only condition participants look at the map to find directly the stories, in the Markers+GPS condition they look at the map to find the markers and to scan them, and in the Full condition they look at the map while listening to the narrator clips, to find the markers and to scan them. Therefore, the additional time that participants spent looking at the screen in the Full condition, can be explained by the observation that participants tend to look at the screen right after the vibration alert, when the narrator audio clip is played, while the amount of time spent looking at the map is similar across conditions.

The statistical analysis also revealed that the total duration of the experience is longest with the Full version, shorter with Markers+GPS, and shorter still with GPS-only (all differences are statistically significant). Across all conditions the duration of the stories playback and the amount of time needed to navigate should be the same. Additionally, in the Markers+GPS condition, as well as in the Full condition, participants needed some additional time to look for the markers in the physical environment (they do this while not looking at the screen). So this activity adds to the total duration of these two conditions. Finally, in the Full condition additional time is spent listening to the narrator, as revealed by the observations.

When we consider the percentage of time that people spend looking at the screen over the total duration of the experience, we see that the percentage was on average lower in the Markers+GPS condition (mean: 58\%) than in the other two conditions (GPS-only mean: $67 \%$, Full mean: $66 \%$ ). The percentage of screen time with Markers+GPS is lower than the GPS-only because the experience takes longer (because of the time to look for the markers), not because people look at the screen less. On the other hand, the percentage of screen time with the full version is higher than Markers+GPS because people look at the screen longer (they look at it when the narrator talks). 
The post-task questionnaire results provide further evidence of the role of the narrator and the markers. In particular, in the Full condition participants reported to find the stories more clearly related to the physical surroundings (as it is desirable) than in the GPS-only condition, while the Markers+GPS condition was not found to differ from either of the other two. Similarly, participants from the Full condition rated the experience as more engaging than those from the GPS-only condition, while the Markers+GPS condition did not differ from either of the other two. These results suggest that the integration of both narrator and markers makes a difference from the user perspective, while markers alone do not seem to cause effects that are as strong. Such results are also reinforced by the additional observations gathered in Study 2. In particular, the additional observations indicate that when the narrator was not present some participants asked the researchers for the connection between stories and surroundings, and some demonstrated confusion about the start and end of the experience. Moreover, it was observed that without the markers, in the GPS-only condition, participants often experienced the stories in locations that differed from those intended.

\subsection{General Discussion}

We discuss the results of the evaluations of Seven Stories (Study 1 and Study 2), pointing out some more general reflections and design implications for location-based cultural applications.

\subsubsection{Visual Markers as Support against GPS Inaccuracy}

One of the main technical issues affecting Seven Stories is the inaccuracy and erratic behaviour of the GPS; this finding is consistent with prior literature about location-based games and cultural applications (Benford et al., 2009; Crabtree et al., 2004) (Rashid et al., 2006). This issue motivated us to redesign the story experience working around the GPS technology failure by adopting a mixed strategy based on analogue literary device of the narrator and the digital one of the visual markers. The findings from Study 1 related to the "orienting function of the narrator" and the "markers as tangible feedback and reward" indicate that the combination of a narrator and the integration of the d-touch markers were successful in helping our participants in orienting themselves and giving them confidence that they were in the correct location. This claim is supported by the results of Study 2: the quantitative results from the participant observation indicate that participants were more inclined to explore when the visual markers were integrated in the Seven Stories experience.

The markers did not make GPS work better, and GPS is still required to trigger the narrator's audio, but participants seemed to be more tolerant of the GPS being slow, because they got the clear, tangible feedback that they were in the right place based on the narrative and the presence of the markers. The integration of the two different positioning techniques, then, produces effects that go beyond the simple replacement of GPS positioning with tagging, as reported for example by Rashid et al (Rashid et al., 2006). As opposed to self-reporting their own position, a strategy adopted by (Benford et al., 2006; 'Uncle Roy All Around You', n.d.), our system left the user feeling supported and guided by the system which would act through the narrator by pointing them in the right direction and to the relevant location. Subsequently upon finding the marker their reassurance would be completed. The audience would not have to leave the story world to take the conscious act of reporting their position, remaining focused on the experience of following the narrator and its stories.

\subsubsection{Aesthetic Function of the Markers}

The use of the d-touch markers was very well-received, both in terms of their visual appeal and for the meaningful role that they played in the interactive experience, elegantly triggering video content once the user had adequately explored the environment (in order to find the marker). In contrast to the use of visual or RFID tags in earlier location-based systems (e.g. (O'Hara et al., 2007a), (Rashid et al., 2006), (O'Hara, 2008) ), our markers are not just machine-readable artefacts, their shape is an integral part of the visual media to convey the content of the stories. 
Yet, their consistent visual language, including their high contrast, makes them easy to recognise as interactive elements. In the light of the above, the function of the markers should be understood through the pragmatist aesthetic interaction paradigm (Petersen et al., 2004), which states that for interactive systems aesthetics needs to be considered beyond form: "it is an integral part of the understanding of an interactive system, and its potential use". Moreover, the markers were also appreciated from the visual point of view, by both participants and nonparticipants. Some users found them "cute" and inspiring. The markers also captivated the attention of local inhabitants not directly involved in the Seven Stories evaluation, in the case of both Study 1 and Study 2. In one case, when some markers fell to the ground, they even inspired care and preservation by some local ladies, who considered them something to be valued.

Deriving from the findings of our evaluation, we argue that markers, and in particular designable ones, can be used instrumentally to foster a pleasurable user experience: the audience has to perform a treasure hunt to search for them, and thus they are encouraged to further explore the surrounding space, and this can be an engaging (Study 2 questionnaire), and rewarding (Study 1 qualitative observations) action. Besides the use of markers with the narrator clues of where they are combines the user's attention and the surrounding space with the trigger of relevant content without disrupting the immersion in the whole experience. According to Peterson, we can call such a pleasure "Aesthetic interaction" (Petersen et al., 2004). Our results show that combined with their visual qualities, such characteristics of the markers can very much enhance user engagement.

\subsubsection{Story Content and Narrator}

Study 2 revealed that with the narrator (only available in the Full condition) participants found the stories more strongly related to the physical surroundings, and reported the experience as more engaging. We argue that such observation indicates that the literary strategy of employing a narrator to guide users, supporting them in the exploration of the narrative space and physical environment, worked well and it generally helpful in delivering a satisfying location-based experience. While the narrator has been extensively used as a literary device in literature (Abbott, 2008), digital narratives and interactive games (Epstein \& Vergani, 2006; Lombardo \& Damiano, 2012), it has never been exploited as a connecting thread between different positioning technologies, like visual markers and GPS.

Our two studies indicate that the narrator is a successful device for guiding the audience through a location-based experience. Its function can also be exploited as a context enhancer, functioning as a trait d'union between different location-based technologies to connect the story content with the physical space where the digital experience takes place. It is worth emphasising that, based on the statistical analysis of the Study 2 questionnaire and additional observations, the addition of visual markers alone did not result in the same effect, despite the high location accuracy that they provide. Participants do not seem to understand the connection between stories and places just by being in the "right spot", the additional explicit explanation provided by the narrator is necessary.

\subsubsection{Physical Context over Digital Content}

In relation to the connection that participants were able to infer between the locations and the stories, some implications for future design can be highlighted. First, we noticed that physical features have predominance over digital ones. For example, the existence of landmarks will affect people's expectations. Hence, when storyboarding and producing the content it is important to incorporate existing landmarks, to take into account how they may influence the designed experience. As mentioned earlier, in Study 1 we observed that very prominent landmarks such as churches or old buildings with a certain look have the power to attract users. Participants felt drawn to landmarks or colourful buildings and started looking for the markers nearby even if the narrator or the mobile interface said otherwise. After a few minutes of searching and not finding what they had expected, they checked the GPS and noticed that their 
location on the map wasn't close to where the icon of the marker was positioned, so they went back to the road that they had left. Such findings highlight an important issue when designing for site-specific experiences: the real space around the users although not part of the designed trajectory of the experiences, strongly influences the trajectory of the users, echoing the findings reported by Benford et. al, in their meta-analysis of various location based experiences (Benford et al., 2009). Despite the mobile interface, markers and narrator guidance, users would still use the physical world as a main reference.

Another important implication for the design of a mobile cultural application is the balance between how much the users look at the screen and how much they enjoy the surrounding landscape. While this finding is not new in itself (Nisi et al., 2006) our Study 2 revealed significant differences across the conditions. The introduction of the markers prompted participants to explore the physical locations where the interactive experience was set, as an off-screen activity. This activity can create a better balance between on-screen and off-screen activities within the experience, a desirable feature for location-based cultural applications. Hence the combination of vibration alerts and markers seems to be a successful strategy to support the transition of user attention from physical features to digital content in location based experiences.

\subsection{Limitations and future work}

Through our two studies we also noted a number of limitations of our system. Firstly, the introduction of the narrator had the effect of drawing the participants attention to the screen for longer. Participants were observed looking at the screen during the playback of the narrator clips, even though these were audio only. It should be noted that during the playback of these clips the screen showed the map, so an opportunity for future work is to assess whether a blank screen, or other visuals could help divert the user's attention from the screen. Secondly, the narrator still manifested some limitations, as some users found it difficult to position themselves in the right spot. Moreover, the use of the narrator pointing out architectural features around the street could have been used more often, it was a feature that was much appreciated by the audience and it was explicitly suggested to us that we use it more often. Thirdly, the narrator anticipating the search for the markers possibly detracted from the engagement in the treasure hunt aspects of the experience. Attention could be placed in preserving the treasure hunt pleasure in location-aware systems like ours, possibly by crafting a narration that entices the search without spoiling it. Finally, the markers themselves, appreciated by users as well as nonusers of the system, could be developed further and exploited more in location-aware cultural experiences. Aesthetically pleasing and visible markers can be used to publicise the experience, by choosing more interesting materials such as ceramic or stone, making them bigger and more colourful, they could become almost self standing artefacts, in line with the Portuguese tradition of ceramic azulejos, the decorative tiles that adorn streets and buildings.

\section{CONCLUSION}

In this paper we have presented the iterative design, implementation and evaluation of Seven Stories, a location-based narrative experience, set on the island of Madeira. Like many other location-based systems, Seven Stories uses GPS to deliver content to users when they are in specific positions in space. However, an initial pilot evaluation of the system revealed some issues related to user orientation and disruption of user immersion in the experience. We then iterated the original design to integrate visual markers and a narrator to refine the experience and help users orient themselves in the physical context.

Building on the analysis of two studies we have highlighted general guidelines and implications for the future of the design of location-based narrative systems. In particular we highlight the importance of a careful design for balanced and fluid user transitions from digital content to the 
physical aspects of the experience, and how technological as well as narrative design solutions can help with this. Moreover we can confirm that the combined use of the two devices, markers and narrator, ameliorated the experience in terms of engagement and relation of the digital content to the physical surroundings (including also more exploration of the physical space). We hope that these findings will help others in designing successful location-based applications and cultural storytelling experiences, and, in general, encourage the comparative evaluations of the specific features of such system.

\section{ACKNOWLEDGMENTS}

Removed for blind review

(ethics approval details)

\section{REFERENCES}

Abbott, H. P. (2008). The Cambridge Introduction to Narrative (2 edition). Cambridge; New York: Cambridge University Press.

Abowd, G. D., Atkeson, C. G., Hong, J., Long, S., Kooper, R., \& Pinkerton, M. (1997). Cyberguide: A mobile context-aware tour guide. Wireless Networks, 3(5), 421-433. http://doi.org/10.1023/A:1019194325861

Angus, A., Papadogkonas, D., Papamarkos, G., Roussos, G., Lane, G., Martin, K., ... Silverstone, R. (2008). Urban Social Tapestries. IEEE Pervasive Computing, 7(4), 44-51.

Aoki, P. M., Grinter, R. E., Hurst, A., Szymanski, M. H., Thornton, J. D., \& Woodruff, A. (2002). Sotto Voce: Exploring the Interplay of Conversation and Mobile Audio Spaces. In Proceedings of the SIGCHI Conference on Human Factors in Computing Systems (pp. 431-438). New York, NY, USA: ACM. http://doi.org/10.1145/503376.503454

Ballagas, R., Kuntze, A., \& Walz, S. P. (2008). Gaming Tourism: Lessons from Evaluating REXplorer, a Pervasive Game for Tourists. In J. Indulska, D. J. Patterson, T. Rodden, \& M. Ott (Eds.), Pervasive Computing (pp. 244-261). Springer Berlin Heidelberg. Retrieved from http://link.springer.com/chapter/10.1007/978-3-540-79576-6_15

Bell, M., Chalmers, M., Barkhuus, L., Hall, M., Sherwood, S., Tennent, P., ... Hampshire, A. (2006). Interweaving Mobile Games with Everyday Life. In Proceedings of the SIGCHI Conference on Human Factors in Computing Systems (pp. 417-426). New York, NY, USA: ACM. http://doi.org/10.1145/1124772.1124835 
Benford, S., Crabtree, A., Flintham, M., Drozd, A., Anastasi, R., Paxton, M., ... Row-Farr, J. (2006). Can You See Me Now? ACM Trans. Comput.-Hum. Interact., 13(1), 100-133. http://doi.org/10.1145/1143518.1143522

Benford, S., Giannachi, G., Koleva, B., \& Rodden, T. (2009). From Interaction to Trajectories: Designing Coherent Journeys Through User Experiences. In Proceedings of the SIGCHI Conference on Human Factors in Computing Systems (pp. 709-718). New York, NY, USA: ACM. http://doi.org/10.1145/1518701.1518812

Braun, V., \& Clarke, V. (2006). Using thematic analysis in psychology. Qualitative Research in Psychology, 3(2), 77-101. http://doi.org/10.1191/1478088706qp063oa

Brown, B., Chalmers, M., Bell, M., Hall, M., MacColl, I., \& Rudman, P. (2005). Sharing the square: Collaborative Leisure in the City Streets. In H. Gellersen, K. Schmidt, M. Beaudouin-Lafon, \& W. Mackay (Eds.), ECSCW 2005 (pp. 427-447). Springer Netherlands. Retrieved from http://link.springer.com/chapter/10.1007/1-4020-4023-7_22

Cater, K., Fleuriot, C., Hull, R., \& Reid, J. (2005). Location Aware Interactive Applications. In ACM SIGGRAPH 2005 Sketches. New York, NY, USA: ACM. http://doi.org/10.1145/1187112.1187133

Chalmers, M., \& Galani, A. (2004). Seamful Interweaving: Heterogeneity in the Theory and Design of Interactive Systems. In Proceedings of the 5th Conference on Designing Interactive Systems: Processes, Practices, Methods, and Techniques (pp. 243-252). New York, NY, USA: ACM. http://doi.org/10.1145/1013115.1013149

Cheverst, K., Davies, N., Mitchell, K., \& Friday, A. (2000). Experiences of developing and deploying a context-aware tourist guide: the GUIDE project. In Proceedings of the 6th annual international conference on Mobile computing and networking (pp. 20-31). ACM. Retrieved from http://dl.acm.org/citation.cfm?id=345916

Ciolfi, L., \& Bannon, L. J. (2007). Designing hybrid places: merging interaction design, ubiquitous technologies and geographies of the museum space. CoDesign, 3(3), 159-180. http://doi.org/10.1080/15710880701524559 
Costanza, E., \& Huang, J. (2009). Designable Visual Markers. In Proceedings of the SIGCHI Conference on Human Factors in Computing Systems (pp. 1879-1888). New York, NY, USA: ACM. http://doi.org/10.1145/1518701.1518990

Crabtree, A., Benford, S., Rodden, T., Greenhalgh, C., Flintham, M., Anastasi, R., ... Steed, A. (2004). Orchestrating a Mixed Reality Game 'on the Ground'. In Proceedings of the SIGCHI Conference on Human Factors in Computing Systems (pp. 391-398). New York, NY, USA: ACM. http://doi.org/10.1145/985692.985742

Crow, D., Davenport, G., \& Pan, P. (2003). System architecture for developing mobile cinema. In CM Multimedia Conference (pp. 2-8). Retrieved from http://mf.media.mit.edu/pubs/conference/ArchMobile.pdf

Epstein, M., \& Vergani, S. (2006). History Unwired: Mobile Narrative in Historic Cities. In Proceedings of the Working Conference on Advanced Visual Interfaces (pp. 302-305). New York, NY, USA: ACM. http://doi.org/10.1145/1133265.1133327

Fitzgerald, E., Taylor, C., \& Craven, M. (2013). To the Castle! A comparison of two audio guides to enable public discovery of historical events. Personal and Ubiquitous Computing, 17(4), 749760. http://doi.org/10.1007/s00779-012-0624-0

Grinter, R. E., Aoki, P. M., Szymanski, M. H., Thornton, J. D., Woodruff, A., \& Hurst, A. (2002). Revisiting the Visit:: Understanding How Technology Can Shape the Museum Visit. In Proceedings of the 2002 ACM Conference on Computer Supported Cooperative Work (pp. 146155). New York, NY, USA: ACM. http://doi.org/10.1145/587078.587100

Haahr, M. (2015). Real World, Real Monsters: Adapting Gothic Horror for Location-Based Augmented-Reality Games.

Hansen, F. A., \& Grønbæk, K. (2008). Social Web Applications in the City: A Lightweight Infrastructure for Urban Computing. In Proceedings of the Nineteenth ACM Conference on Hypertext and Hypermedia (pp. 175-180). New York, NY, USA: ACM. http://doi.org/10.1145/1379092.1379126 HearUsHere Team. (n.d.). HearUsHere. Retrieved from http://www.hearushereapp.com/ 
Lombardo, V., \& Damiano, R. (2012). Storytelling on mobile devices for cultural heritage. New Review of Hypermedia and Multimedia, 18(1-2), 11-35. http://doi.org/10.1080/13614568.2012.617846

Løvlie, A. S. (2012). You are the one thinking this: locative poetry as deictic writing. New Review of Hypermedia and Multimedia, 18(1-2), 109-127. http://doi.org/10.1080/13614568.2012.617845 Lund, K., Coulton, P., \& Wilson, A. (2011). Free All Monsters!: a context-aware location based game (p. 675). ACM Press. http://doi.org/10.1145/2037373.2037482

Lynch, K. (1960). The Image of the City. MIT Press.

Magyaródi, T., Nagy, H., Soltész, P., Mózes, T., \& Oláh, A. (2013). Psychometric properties of a newly established flow state questionnaire. In The Journal of Happiness \& Well-Being (Tayfun DOĞAN, Ph.D, Vol. 1, pp. 89-100).

Millard, D. E., \& Hargood, C. (2015). A Research Framework for Engineering Location-Based Poetics (pp. 13-16). ACM Press. http://doi.org/10.1145/2804565.2806671

Nisi, V., Oakley, I., \& Haahr, M. (2006). Inner City Locative Media: Design and Experience of a Location-Aware Mobile Narrative for the Dublin Liberties Neighborhood. In Intelligent Agent (Vol. 6). Retrieved from http://mf.media.mit.edu/pubs/journal/InnerCity.pdf

Nisi, V., Oakley, I., \& Posthuma De Boer, M. (2010). Locative Narratives as Experience: a new perspective on Location Aware Multimedia Stories. Presented at the ArTech 2010, Porto, Portugal.

Nisi, V., Wood, A., Davenport, G., \& Oakley, I. (2004). Hopstory: An Interactive, Location-Based Narrative Distributed in Space and Time. In S. Göbel, U. Spierling, A. Hoffmann, I. lurgel, O. Schneider, J. Dechau, \& A. Feix (Eds.), Technologies for Interactive Digital Storytelling and Entertainment (pp. 132-141). Springer Berlin Heidelberg. Retrieved from http://link.springer.com/chapter/10.1007/978-3-540-27797-2_18

O'Hara, K. (2008). Understanding Geocaching Practices and Motivations. In Proceedings of the SIGCHI Conference on Human Factors in Computing Systems (pp. 1177-1186). New York, NY, USA: ACM. http://doi.org/10.1145/1357054.1357239 
O'Hara, K., Kindberg, T., Glancy, M., Baptista, L., Sukumaran, B., Kahana, G., \& Rowbotham, J. (2007a). Collecting and Sharing Location-based Content on Mobile Phones in a Zoo Visitor Experience. Computer Supported Cooperative Work (CSCW), 16(1-2), 11-44. http://doi.org/10.1007/s10606-007-9039-2

O’Hara, K., Kindberg, T., Glancy, M., Baptista, L., Sukumaran, B., Kahana, G., \& Rowbotham, J. (2007b). Social Practices in Location-based Collecting. In Proceedings of the SIGCHI Conference on Human Factors in Computing Systems (pp. 1225-1234). New York, NY, USA: ACM. http://doi.org/10.1145/1240624.1240810

Parkes, A., \& Ängeslevä, J. (2007). Physical interventions in a location based cultural narrative: a case study of embedded media in public space installations (p. 65). ACM Press. http://doi.org/10.1145/1226969.1226982

Petersen, M. G., Iversen, O. S., Krogh, P. G., \& Ludvigsen, M. (2004). Aesthetic Interaction: A Pragmatist's Aesthetics of Interactive Systems. In Proceedings of the 5th Conference on Designing Interactive Systems: Processes, Practices, Methods, and Techniques (pp. 269-276). New York, NY, USA: ACM. http://doi.org/10.1145/1013115.1013153

Rashid, O., Bamford, W., Coulton, P., Edwards, R., \& Scheible, J. (2006). PAC-LAN: mixedreality gaming with RFID-enabled mobile phones. Computers in Entertainment, 4(4), 4. http://doi.org/10.1145/1178418.1178425

Reid, J., Hull, R., Cater, K., \& Fleuriot, C. (2005). Magic Moments in Situated Mediascapes. In Proceedings of the 2005 ACM SIGCHI International Conference on Advances in Computer Entertainment Technology (pp. 290-293). New York, NY, USA: ACM. http://doi.org/10.1145/1178477.1178529

Rogers, Y. (2011). Interaction Design Gone Wild: Striving for Wild Theory. Interactions, 18(4), 5862. http://doi.org/10.1145/1978822.1978834

Schilit, B. N., Adams, N., Gold, R., Tso, M. M., \& Want, R. (1993). The PARCTAB mobile computing system. In , Fourth Workshop on Workstation Operating Systems, 1993. Proceedings (pp. 34-39). http://doi.org/10.1109/WWOS.1993.348175 
Siira, E., Tuikka, T., \& Tormanen, V. (2009). Location-Based Mobile Wiki Using NFC Tag Infrastructure. In First International Workshop on Near Field Communication, 2009. NFC '09 (pp. 56-60). http://doi.org/10.1109/NFC.2009.8

Steed, A. (2004). Supporting Mobile Applications with Real-Time Visualisation of GPS Availability. In S. Brewster \& M. Dunlop (Eds.), Mobile Human-Computer Interaction - MobileHCI 2004 (pp. 373-377). Springer Berlin Heidelberg. Retrieved from http://link.springer.com/chapter/10.1007/978-3-540-28637-0_40

Stevens, Q. (2007). The Ludic City: Exploring the Potential of Public Spaces. Routledge. Team 7scenes. (n.d.). 7scenes. Retrieved from http://7scenes.com/

Timothy Sanders, P. C. (2010). Time perception, immersion and music in videogames., 160-167. Uncle Roy All Around You: Implicating the City in a Location-Based Performance | Steve Benford, Martin Flintham, Adam Drozd, Rob Anastasi, Duncan Rowland, Nick Tandavanitj, Matt Adams, Ju Row-Farr, Amanda Oldroyd, Jon Sutton. (n.d.). Retrieved 3 July 2015, from http://www.europeana.eu/portal/record/2022113/urn_axmedis_00000_obj_a5e61424_e3f1_46ea _b471_99a9536a5a73.html

van Leeuwen, J. P., Karnik, M., \& Keane, K. (2011). Discovering Madeira: A Case Study of Cultural Probes. In Procedings of the Second Conference on Creativity and Innovation in Design (pp. 439-447). New York, NY, USA: ACM. http://doi.org/10.1145/2079216.2079282

Verplank, B. (n.d.). Interaction design sketchbook. Retrieved from https://hci.rwth-aachen.de/tikidownload_wiki_attachment.php?attld=797

Vriesede, T., \& Nack, F. (2011). StoryStream: Unrestricted Mobile Exploration of City Neighbourhoods Enriched by the Oral Presentation of User-Generated Stories. In M. Si, D. Thue, E. André, J. C. Lester, J. Tanenbaum, \& V. Zammitto (Eds.), Interactive Storytelling (Vol. 7069, pp. 231-242). Berlin, Heidelberg: Springer Berlin Heidelberg. Retrieved from http://link.springer.com/10.1007/978-3-642-25289-1_25

Want, R., Hopper, A., Falcão, V., \& Gibbons, J. (1992). The Active Badge Location System. ACM Trans. Inf. Syst., 10(1), 91-102. http://doi.org/10.1145/128756.128759 
Wood, R. T. A., Griffiths, M. D., \& Parke, A. (2007). Experiences of Time Loss among Videogame Players: An Empirical Study. CyberPsychology \& Behavior, 10(1), 38-44. http://doi.org/10.1089/cpb.2006.9994 